\title{
SECURE MULTIPARTY COMPUTATION
}

\author{
Renren Dong
}

\author{
A Thesis \\ Submitted to the Graduate College of Bowling Green \\ State University in partial fulfillment of \\ the requirements for the degree of \\ MASTER OF SCIENCE \\ August 2009 \\ Committee: \\ Ray Kresman, Advisor \\ Mohammad Dadfar \\ So-Hsiang Chou
}




\begin{abstract}
Ray Kresman, Advisor

Data mining algorithms help reveal hidden information in a repository. Distributed mining algorithms meet this need by distributing data and computation. One of the most important issues of these algorithms is how to safely mine the data. Secure Multiparty Computation (SMC), a framework for safe mining of distributed data, provides some security promises of the computation.

This thesis addresses certain aspects of SMC including the role of Hamiltonian and edgedisjoint Hamiltonian cycles. We formalize the notion of trust in a network and show that certain network configurations are better than others. We propose and analyze an algorithm for id assignment in networks that outperforms an existing algorithm.
\end{abstract}




\section{Table of Contents}

$\begin{array}{ll}\text { CHAPTER 1: INTRODUCTION } & 1\end{array}$

1.1 Data Mining . . . . . . . . . . . . . . . . . . . . . . . . 1

1.1.1 What is data mining? . . . . . . . . . . . . . . . . . . 1

1.1 .2 Association rule mining algorithms . . . . . . . . . . . . . . . . . . 3

1.1 .3 Distributed data mining . . . . . . . . . . . . . . . . . . . . 5

1.1 .4 Privacy-preserving data mining . . . . . . . . . . . . . . . . 6

1.1.5 Secure multiparty computation . . . . . . . . . . . . . . . 7

1.2 Graph Theory . . . . . . . . . . . . . . . . . . . . . . . 9

1.2 .1 Introduction and definition . . . . . . . . . . . . . . . . . . 9

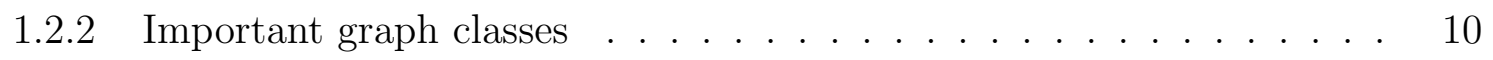

$1.2 .3 \quad$ Hamiltonian cycle and edge-disjoint Hamiltonian cycle . . . . . . . . 10

1.3 Our Work $\ldots \ldots \ldots \ldots$. . . . . . . . . . . . . . . . . . . . . . . . . . . . . . . . . . 12

CHAP TER 2: EDGE-DISJOINT HAMILTONIAN CYCLE 13

2.1 Hamiltonian Cycle $\ldots \ldots \ldots \ldots$. . . . . . . . . . . . . . . 13

2.1 .1 Basic Hamiltonian cycle theorems . . . . . . . . . . . . . . . . . . . . 14

2.1 .2 Hamiltonian cycle and random graph . . . . . . . . . . . . . . . . . 14

$2.1 .3 \quad$ Overview of Hamiltonian cycle algorithms . . . . . . . . . . . . . . . 15

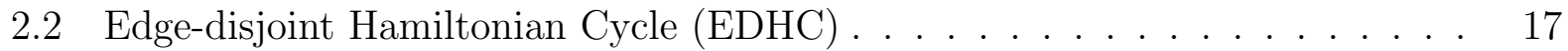

2.3 Order of Discovering EDHC $\ldots \ldots \ldots \ldots$ 
2.4 Heuristic Algorithms for EDHC . . . . . . . . . . . . . . . . . . 23

2.4 .1 Heuristic dynamic local search - EDHC . . . . . . . . . . . . . 25

CHAPTER 3: TRUST ENABLED SECURE MULTIPARTY COMPUTA-

$\begin{array}{ll}\text { TION } & 30\end{array}$

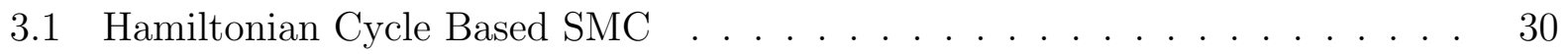

3.2 Notion of Trust . . . . . . . . . . . . . . . . . . . . . . . . . . . . . . . 32

3.2 .1 Defend against collusion attack . . . . . . . . . . . . . . . . . . . . 33

3.3 A Model for Trust Enabled HSMC (TE-HSMC) . . . . . . . . . . . . . . . . 34

3.4 Greedy Algorithm for TE-HSMC . . . . . . . . . . . . . . . . . . . . . . . . 37

3.4 .1 Experimental results . . . . . . . . . . . . . . . . . . . 37

CHAPTER 4: ANONYMOUS OPT-OUT AND ID ASSIGNMENT 40

4.1 Anonymous Opt-Out . . . . . . . . . . . . . . . . . . . . 40

$4.1 .1 \quad$ Overview of Dining Cryptographers (DC) problem . . . . . . . . . . . 41

4.1 .2 Anonymous opt-out in CPSS . . . . . . . . . . . . . . . . . . 43

4.2 Anonymous ID $\ldots \ldots \ldots \ldots$

$4.2 .1 \quad$ Anonymous ID assignment using SS . . . . . . . . . . . . . . . . . . 45

4.2 .2 Analysis of anonymous ID algorithms . . . . . . . . . . . . . . . . . 49

CHAPTER 5: CONCLUDING REMARKS 59

5.1 Contribution . . . . . . . . . . . . . . . . . . . . . . 59

5.2 Future Work . . . . . . . . . . . . . . . . . . . . . . . 60

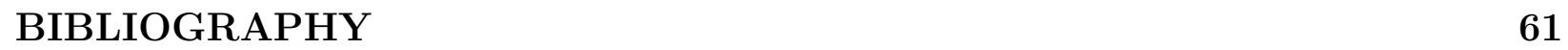




\section{List of Figures}

1.1 Secure Sum . . . . . . . . . . . . . . . . . . . . 8

1.2 Important Graphs $\ldots \ldots \ldots \ldots 11$

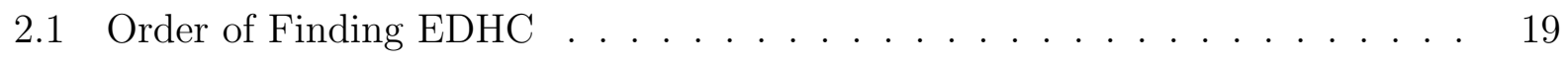

$2.2 \quad$ Another Order of Finding EDHC $\ldots \ldots \ldots \ldots$

$2.3 \quad$ Maximum Clique of Size 3 . . . . . . . . . . . . . . . . . . . . . . 21

2.4 Transform MC to REDC . . . . . . . . . . . . . . . . . . . . . . . . . . 22

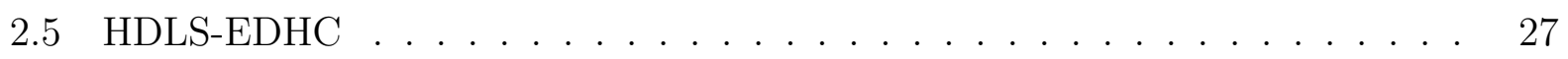

2.6 HDLS-EDHC-expandSearch . . . . . . . . . . . . . . . . . 28

2.7 HDLS-EDHC - plateauSearch $\ldots \ldots \ldots \ldots \ldots$

3.1 Secure Sum Computation - One Cycle $\ldots \ldots \ldots$

$3.2 \quad$ Secure Sum Computation - Two Cycles $\ldots \ldots \ldots$

3.3 Configuration of SMC $\ldots \ldots \ldots \ldots \ldots \ldots$

$3.4 \quad$ An Improved Configuration of SMC $\ldots \ldots \ldots \ldots$

3.5 Safety Level of Node $\mathrm{A} . \ldots \ldots \ldots$

3.6 Safety Level with CPSS $\ldots \ldots \ldots$

$3.7 \quad$ Swap Operation $\ldots \ldots \ldots \ldots$

$4.1 \quad$ Illustration of DC-Net $\ldots \ldots \ldots \ldots$. . . . . . . . . . . . . . . . . 42

4.2 CPSS with Opt-Out (None Opts-out) $\ldots \ldots \ldots$. . . . . . . . . . 44

4.3 CPSS with Opt-Out (Someone Opts-out) $\ldots \ldots \ldots$. . . . . . . . . . 44 
4.4 Basic Anonymous ID Assignment (AIDA-A), $m=3, n=3$. . . . . . . . 47

4.5 Modified Anonymous ID Assignment (AIDA-B), $m=6, n=3$. . . . . . . 49

4.6 Mean Number of IDs Assigned in a Round (AIDA-A), $0 \leq M \leq 50, N \leq 30 \quad 53$

$4.7 \quad$ Comparison of $A p$ Expected $_{(A I D A-A)}(M, N)$ and $\operatorname{Expected}_{(A I D A-A)}(M, N)$. . 54

4.8 Mean Number of IDs Assigned in a Round (AIDA-B), $0 \leq M \leq 50, N \leq 30 . \quad 56$

4.9 Approximate Evaluation of Mean Number of Rounds (AIDA-A and AIDA-B), $0 \leq M \leq 50, N \leq 30 \ldots \ldots$. . . . . . . . . . . . . . . . 58 


\section{List of Tables}

3.1 A Trust Matrix . . . . . . . . . . . . . . . . . . . . . . . . . . . . . . 35

3.2 Synthetic Workload and Swap Improvement . . . . . . . . . . . . . . . . . . 38

3.3 Performance of Swap Improvement . . . . . . . . . . . . . . . . . . . . . . . 39 


\section{CHAPTER 1}

\section{INTRODUCTION}

\subsection{Data Mining}

In the past decade, data mining has become an important tool to help people discover knowledge hiding in the data. As well, mining data in a secure fashion has been a valuable research domain. We introduce some basic ideas related to data mining and secure data mining in this section.

\subsubsection{What is data mining?}

Data mining is the process of extracting hidden patterns from data. As more and more data is collected all over the world, data mining is becoming an increasingly important tool to transform the data into knowledge. In the business world, data mining is a powerful new technology with potential to help companies focus on the hidden-intelligence in their

data warehouses. Data mining can be used to predict future trends and behavior allowing businesses to make proactive decisions. One wildly used example of data mining is to mine the historical transaction data in a store. One could mine a set of retail transactions from a supermarket to generate the sale patterns. For example, the purchase pattern could lead to the knowledge discovery that purchase of office chairs is often associated with the purchase 
of fancy staplers. By discovering this pattern, one could improve the sales by co-locating office chairs and fancy staplers.

In order to discover valuable information, the field of data mining provides techniques for new knowledge discovery - finding patterns in the data set such as classifiers and association rules that are useful to the data miner. We will only consider association rules in this thesis. The association rule is a method for discovering interesting and valuable relationships between items or variables in large databases. An example of an association rule in store data mining is $\{$ onions,potatoes $\} \rightarrow\{$ beef $\}$ which indicates that the purchase of onions and potatoes is often associated with the purchase of beef. Association rules are employed today in many application areas including web usage mining, intrusion detection and bioinformatics [42] [28].

The definition of association rules is based on two statistical parameters: support and confidence. Support is a measure of how frequently a given set of items appears in the database (DB). Confidence, on the other hand, is a conditional probability; that is, given a set of items, what is the likelihood another disjoint set of items will be in the same item set. Let $I$ be the set of all items in the database $D$. The database $D$ may be thought of as a collection of records, $D=\cup_{i=1}^{N} R_{i}, R_{i} \subseteq I$, where $N$ is the size of DB. Let us consider two itemsets $A, B \subseteq I$. An association rule is defined as $A \rightarrow B$, indicates that $B$ is likely whenever $A$ is likely. A formal definition of support and confidence follows. The set $A \cup B$ has support if counting over all records in $D$ the probability $P(A \cup B)=\frac{\operatorname{Count}(A \cup B)}{N} \geq \operatorname{MinSupport}$ where the parameter MinSupport $\in[0,1]$ is supplied by the data miner [25]. Confidence of rule $A \rightarrow B$ is measured by the conditional probability $P(B \mid A)=\frac{\operatorname{Count}(A \cup B)}{\operatorname{Count}(A)} \operatorname{counting}$ over all records in D [25]. Just as the definition of support, set $A \cup B$ has confidence if $P(B \mid A) \geq$ MinConfidence where the parameter MinConfidence $\in[0,1]$ is also provided by the data miner.

Data mining using association rules, called association rule mining (ARM), is quite powerful and many ARM techniques have been developed in a variety of areas [25]. So it should 
not surprise the reader that algorithms involved in data mining are also somewhat varied. In the next section, we will briefly introduce some well-known ARM algorithms.

\subsubsection{Association rule mining algorithms}

The goal of ARM algorithms is to find association rules. In order to reach this goal, support and confidence value of all the candidate itemsets must be calculated. Here, itemsets of size $k$ is called $k$-itemsets. As a convention, we also denote the set of frequent $k$-itemsets as $L_{k}$ and the set of candidate $k$-itemsets as $C_{k}$. Of course, $1 \leq k \leq N$ where $N$ is the number of distinct items in the database.

One of the best-known algorithms to mine association rules is the Apriori algorithm [2]. It uses a breadth-first search strategy to count the support of itemsets and uses a candidate generation function which exploits the downward closure property of support. The basic idea of Apriori algorithm is that if a $k$-itemset is non-frequent then all supersets of that set will also be non-frequent. This means if we already have $L_{k}$ from the current iteration and we try to generate a new candidate itemset $C_{k+1}$, the only possible candidates we need to consider is the superset of $L_{k}$. Here is how the Apriori algorithm works: (1) count all frequent 1-itemsets to obtain $L_{1} ;(2)$ use $L_{1}$ to generate $C_{2} ;(3)$ count all 2-itemsets in $C_{2}$ and retain frequent ones in $L_{2}$; (4) repeat steps 2 and 3 until all frequent itemsets are counted, that is, obtain $L_{k+1}$ by counting $C_{k+1}$ which in turn is generated from $L_{k}$.

Since Apriori, many improvements have been made in order to speed up the generation of candidate itemsets $C_{k+1}$. Clearly, in order to be efficient, the heuristic should generate candidates that have a high likelihood of being frequent itemsets because for each candidate, we need to count its occurrences in all transactions.

An effective hash-based method called direct hashing and pruning (DHP) was introduced to Apriori by Pack [35]. This algorithm uses hash counts to filter the candidates. Also, the generation of smaller candidate sets enables DHP to effectively trim the transaction database. Transaction reduction is another method to improve Apriori's efficiency. The idea 
of transaction reduction is to remove any $(k+1)$-itemsets that do not contain any frequent $k$-itemsets.

A partitioning technique can also be used to improve Apriori's performance [25]. Partitioning consists of two phases. In phase one, the algorithm divides the DB into $n(n \geq 1)$ non-overlapping partition. If the minimum support threshold for transactions in DB is minSup, then the minimum support count for a partition is minSup $\times$ the number of transactions in that partition. In each partition, all frequent itemsets within that partition, which is also called local frequent itemsets, are found. A local frequent itemset may or may not be frequent with respect to the entire database. So the global candidate itemsets, which is the union of all the local frequent itemset, needs to be calculated. Because all the frequent itemset with respect to the entire database must be frequent in at least one partition, all the global frequent itemset will be in the global candidate itemsets. In phase two, a second scan of all the partitions is conducted to find the actual support count for the global candidate itemsets.

Though a lot of work has been done to improve Apriori, it can still generate a huge number of candidate sets or may still need to repeatedly scan the database and check a large set of candidates. Han [26] discusses a method for generating frequent itemsets without candidate set generation by using a frequent-pattern tree (FP-tree). The basic idea of this method is to compress a large database into a compact, FP-tree structure. FP-tree is highly condensed, but complete for frequent pattern mining and avoids costly database scans. However, one possible drawbacks of FP-tree method is that in some cases the FP-tree could be too large to fit the whole structure into the main memory. In order to solve this problem, a simple and efficient algorithm was developed in [12]. The algorithm uses pre-processing and recursive elimination. It employs singly linked lists instead of an FP-tree, eliminates the need to rerepresent the database records. It is effective in time versus Apriori, and in memory usage versus the FP-tree algorithm.

We have reviewed some ARM algorithms. In the next section, we will discuss ARM 
algorithms for distributed environments.

\subsubsection{Distributed data mining}

ARM algorithms discover association rules by mining a single site or database. However, more and more data today are spread out in multiple databases as in distributed system. Distributed data mining algorithms (DDM) are developed to solve data mining problems in a distributed environment.

Agrawal and Shafer [2] discuss a parallel algorithm called Count Distribution (CD) for mining association rules. The algorithm uses the Apriori algorithm locally at each data site. In each phase, $K$, of the algorithm each site generates $k$-itemsets candidate based on the globally frequent itemset of the prior phase. Then each site calculates the local support counts of the global frequent candidate and broadcasts them to the other sites, and each site can compute the frequent $k$-itemsets based on the global counts.

Another algorithm that is based on Apriori is the distributed mining of association rules [17], also called as Fast Distributed Mining of association rules (FDM/DMA). FDM/DMA generates a smaller number of candidate itemsets then CD, by pruning at each site itemsets that are not locally frequent. Note that an itemset cannot be globally large if it is not locally frequent in at least one site.

The Optimized Distributed Association rule Mining (ODAM) algorithm [5] follows the paradigm of CD and DMA. ODAM is concerned with reducing the communication cost. From the communication point of view, it minimizes the total message exchange by sending support counts of candidate itemsets to a single site called receiver. The receiver will broadcast the globally frequent itemsets back to the distributed sites. At the local mining level, it deletes the items that weren't found in the previous step and also removes duplicate transactions.

DDM algorithms provide us an effective way to mine data in a distributed environment by extending traditional data mining algorithms. One of the problems of DDM is confidentiality. We consider a scenario where two parties having private databases wish to compute the union 
of their databases. Since the databases are confidential, neither party is willing to expose the content of its database to the other. How do we mine the knowledge without divulging any confidential information? Privacy-preserving data mining (PPDM) is a technique that seeks to address just such an issue.

\subsubsection{Privacy-preserving data mining}

A key problem that arises in DDM is confidentiality. The need of privacy in data mining is justified by various reasons. Sometimes it is due to law, sometimes it is motivated by business interests. One could argue that one can gain useful information, as in medical field, by pooling data for research. However, it is often not possible due to the confidentiality issues which arise in the mining process. Privacy-preserving data mining (PPDM) addresses this question.

In order to understand the mechanics of PPDM, we need to understand what privacy in data mining is. Generally, the privacy technique in data mining must ensure that any information disclosed cannot be traced to an individual or does not constitute an intrusion. A formal definition of privacy in data mining is still an open challenge.

Most methods for privacy computations use some form of transformation on the data in order to perform privacy preservation. The essence of PPDM technique is the trade-

off between information loss and privacy. We mention a couple of techniques here. The randomization method [1] is a technique for privacy-preserving data mining in which noise is added to data in order to mask the attribute values of records. The $k$-anonymity model [34] was developed to protect individual identifiability. In the $k$-anonymity method, the authors reduce the granularity of data representation sufficiently to prevent any individually identifiable information from being leaked.

Many more PPDM algorithms exist. A good overview of the state-of-the-art of PPDM is given in [47]. 


\subsubsection{Secure multiparty computation}

Secure multiparty computation (SMC) is the basic operation in PPDM. SMC was first introduced in [50]. The basic idea of SMC is that a computation is secure if at the end of the computation, no party knows anything except its own input and the results. SMC is the basis for PPDM and implementing SMC efficiently and effectively is critical to the performance PPDM.

Before we introduce an example of SMC, two terms which will be useful in the following discussion will be introduced. Horizontally partitioned data and vertically partitioned data are terms used to describe how the data is stored in a distributed system. In a horizontal partition, all the transactions are distributed among multiple sites. The different sites may have different set of records containing the same attributes. On the other hand, in vertical partition, the different sites may have different attributes of the same sets of records.

A number of SMC algorithms are discussed in [18]. These algorithms are the basic building blocks of PPDM. Some examples of these algorithms are given below.

- Secure Sum (SS) [38: The goal of SS is that the value of each site's individual input be masked while the global sum of all inputs is universally known. This means that at the end of the compuation, no party knows anything except its own input and the global sum. Assume we have $\mathrm{N}$ sites. For each site we have a value $V_{i}, 1 \leq i \leq N$ and we want to calculate global sum $V=\sum_{i=1}^{N} V_{i}$.

In Figure 1.1, a possible solution for the secure sum problem is illustrated. The algorithm starts with station $\mathrm{S} 1$ that generates a random number $R=5$. Then $\mathrm{S} 1$ sends $V_{1}+R=8$ to the next station. After $\mathrm{S} 2$ receives $V_{1}+R$, it adds its local value to the sum it received before sending it to the next station. The next site will repeat this process until the circuit closes. When the circuit does close, the start node, S1, will

receive $R+\sum_{i=1}^{N} V_{i}$. Since $\mathrm{S} 1$ knows $R$, the global sum can be easily calculated. SS is important because it is a very frequently used operation in PPDM; for example, the 


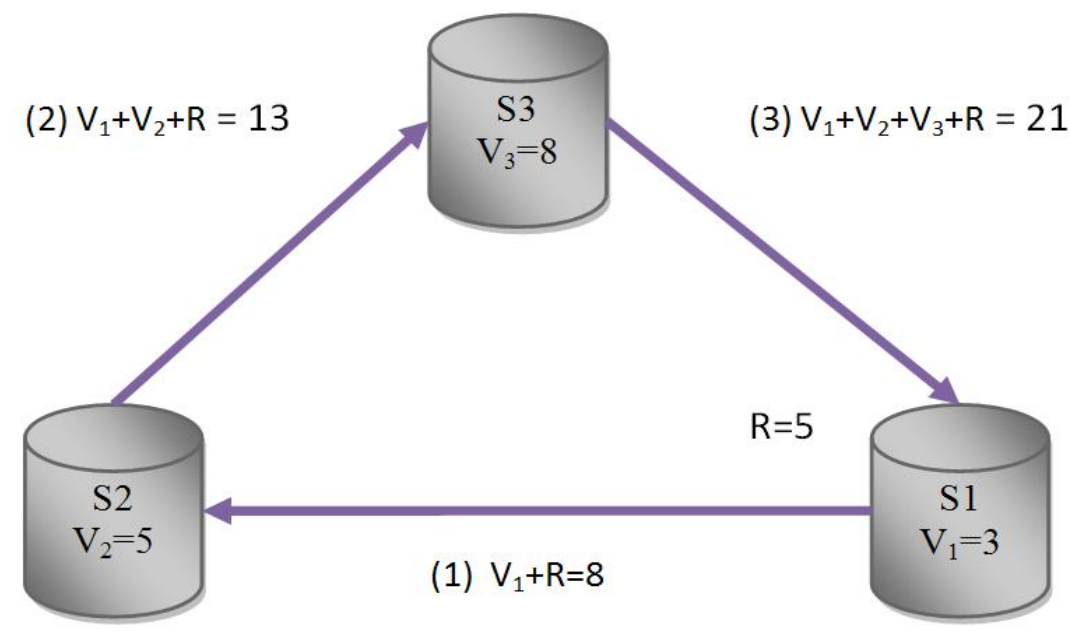

(4) Support $=V_{1}+V_{2}+V_{3}+R-R=16$

Figure 1.1: Secure Sum

$V_{i}$ in Figure 1.1 can be considered as local support count of an itemset, and the global sum, 16, can be treated as global support count of the itemset.

- Secure Set Union: It computes the union of all the local sets without revealing any individually identifiable information. Formally, given $N$ sites, each site has a local set $S_{i}, 1 \leq i \leq N$. We wish to securely compute $\cup_{i=1}^{N} S_{i}$. In PPDM, secure set union methods can be used to generate the global candidate itemset by calculating the union of local frequent itemsets.

- Secure Set Intersection: Consider several parties having their own set of items from a common domain. One problem in PPDM is to securely compute the cardinality or the the intersection of these local sets. Formally, given $N$ sites, each site has a local set $S_{i}, 1 \leq i \leq N$ we wish to securely compute $\cap_{i=1}^{N} S_{i}$.

- Scalar Product: Scalar product is quite powerful and many data mining problems can be reduced to computing the scalar product especially in a vertical partitioned database. The problem can be formally defined as follows: assume we have two sites $\mathrm{S} 1$ and $\mathrm{S} 2$, and each site has a vector of cardinality $n$; $\mathrm{S} 1$ has $\vec{X}=\left(x_{1}, \ldots, x_{n}\right)$ and 
S2 has $\vec{Y}=\left(y_{1}, \ldots, y_{n}\right)$. The problem is to compute the scalar product of the two vecotors, i.e., $\sum_{i=1}^{N}\left(x_{i} \times y_{i}\right)$.

We can see that SMC is an elegant and important tool in PPDM. Having elucidated the basic concepts of ARM, DDM and PPDM, we next turn to some basic graph theory and related algorithms which we found to be helpful in our work.

\subsection{Graph Theory}

Because of the importance of graph theory, we introduce some basic ideas of graph theory and related algorithms in order to help the reader understand our work. Hamiltonian cycles and edge-disjoint Hamiltonian cycles will also be discussed in the later part of this section.

\subsubsection{Introduction and definition}

A graph $G$ is defined by a set $V(G)$ of elements called vertices, a set $E(G)$ of elements called edges. So a graph can be represent by $G=(V, E)$, where $V=V(G)$ and $E=E(G)$. Our work is concerned only with finite graphs, or sets $V(G)$ and $E(G)$ are both finite. An edge $x, x \in E$, can be denoted as an ordered pair $x=(a, b)$ where $a, b \in V$. For an edge $(u, v)$, we use a shorter notation $u v$ to represent the edge. A graph in which edges have no orientation is called undirected graph, otherwise the graph is called directed graph. The number of vertices in a graph is denoted $|V|$ and the number of edges is $|E|$. The degree of a vertex

is the number of edges that connect to it. Vertices $u, v \in V(G)$ are said to be adjacent if $u v \in(G)$. In graph theory, if a number (weight) is assigned to each edge, the graph is called weighted graph. Such weights might represent, for example, cost, lengths or capacities, etc. depending on the problem. The weight of the graph is the sum of the weights of all edges.

A path is defined as a sequence of edges where any two adjacent edges share at least one node. For example, we have path $P_{0}=\left(x_{1}, x_{2}, \ldots, x_{n}\right)$ where, for any fixed $i, 1 \leq i \leq N$, 
$x_{i} \neq x_{i+1}, \exists v \in V$, such that $v \in x_{i} \cap x_{i+1}$. A path, which has $x_{1} \neq x_{N}$ and $\exists v \in V$, $v \in x_{1} \cap x_{N}$, is called cycle.

A graph is bipartite if its vertices can be partitioned into two sets so that all edges have one endpoint in each set. A graph is called $k$-vertex-connected or $k$-edge-connected if removal of $k$ or more vertices (respectively, edges) makes the graph disconnected. A $k$-vertex-connected graph is often called simply $k$-connected [20].

\subsubsection{Important graph classes}

A regular graph is a graph where each vertex has the same degree. A regular graph with vertices of degree $\mathrm{k}$ is called k-regular graph or regular graph of degree k. Figure 1.2(a) is an example of a 2-regular graph.

Complete graph, also known as a connected graph is a type of graph in which that each pair of vertices has an edge connecting them. Figure $1.2(\mathrm{~b})$ is an example of a complete graph with 4 vertices.

A type of graph which is widely used in interconnection network is the $K$-ary $N$-cube. In our work, we will consider torus as a synonym for a $K$-ary $N$-cube. In a $K$-ary $N$-cube, $N$ is the dimension of the graph and $K$ is the number of nodes on each edge. Figure 1.2(c) is an example of a 3-ary 2-cube.

Another type of graph is a grid graph. A grid graph is a graph whose vertices lie on integer coordinates and whose edges connect all pairs of vertices that are unit distance apart. Figure $1.2(\mathrm{~d})$ is an example of a grid graph.

\subsubsection{Hamiltonian cycle and edge-disjoint Hamiltonian cycle}

Hamiltonian cycles (HC) plays an important role in graph theory. A Hamiltonian cycle, $H$, is a cycle that visits every node in the graph exactly once before returning to the source node. Alternatively, a Hamiltonian cycle can be described as a sequence of edges where any two adjacent edges share exactly one unique node in the graph, where the first and 


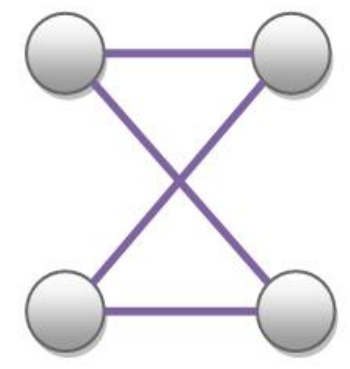

(a)

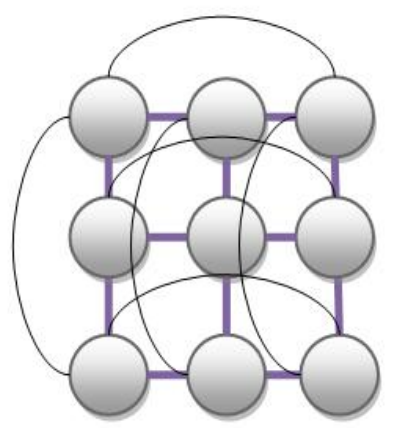

(c)

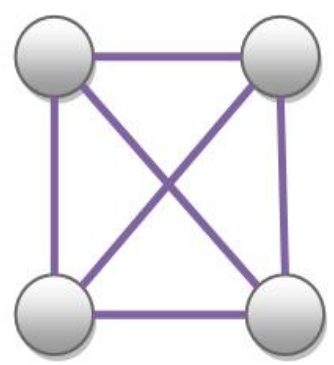

(b)

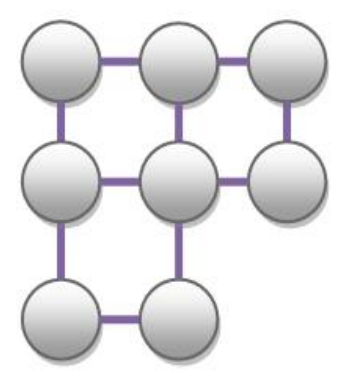

(d)

Figure 1.2: Important Graphs

last edge in the cycle share exactly one unique node in the graph, and where the number of edges in $\mathrm{H}$ equals the number of nodes in the graph. In graph $G=(V, E)$, if we define Hamiltonian cycle as $H=\left\{h_{1}, h_{2}, \ldots, h_{N}\right\}, h_{i} \in E, 1 \leq i \leq N$, we will have $N=|V|$, and $V=\left(\cup_{i=1}^{N-1} h_{i} \cap h_{i+1}\right) \cup\left(h_{N} \cap h_{i}\right)$.

Now we define an edge-disjoint Hamiltonian cycle (EDHC). In a graph $G$, a set of Hamiltonian cycles is called edge-disjoint if every edge in one Hamiltonian cycle is disjoint with every edge in the rest of the Hamiltonian cycles of the set. Formally, suppose we have a set $H=\left\{H^{(i)} \mid H^{(i)}\right.$ is a Hamilton cycle in graph $\left.G\right\},|H|$ is the size of $H$. $H$ is edge-disjoint if $\forall e \in H^{(i)}, e \neq d, \forall d \in H^{(j)}, 1 \leq i \neq j \leq|H|$.

EDHC is useful in computer networks. It can be used to improve the capacity of the network or to provide fault-tolerance and computer security. In our research, we will appeal to the fault tolerance aspects of EDHC. 


\subsection{Our Work}

Our work concerns secure multiparty computation. We seek to improve the security and/or the computational aspects of certain components of SMC.

Chapter 2 extends the discussion of HC and EDHC. Following an introduction to certain well-known results, we look at whether the order in which Hamiltonian cycles are found has an impact on the number of EDHC in the graph. We prove that the problem of finding the largest edge-disjoint cycle set is NP-Complete, and construct a heuristic algorithm to generate EDHC. Chapter 3 introduces the notion of trust between nodes of the network, and looks at its impact on preserving privacy of individual data items. We provide a formal model for computing the mean trust factor of the network as a whole, and design an algorithm that seeks to improve this mean value by using Hamiltonian cycles in which trusted parties are in close proximity. Chapter 4 is devoted to the study of algorithms for assigning anonymous IDs to nodes, and for allowing a node to opt-out of a mining computation if it fears that its privacy can't be preserved - all without allowing anyone to know who, if any, may have opted out. Our ID assignment algorithm improves upon an existing algorithm. We also provide an approximate analysis of the number of rounds needed by the algorithms to finish ID assignment. Concluding remarks are given in Chapter 5 . 


\section{CHAPTER 2}

\section{EDGE-DISJOINT HAMILTONIAN CYCLE}

Hamiltonian cycle (HC), a well known NP-Complete problem, involves finding a cycle in a given graph containing all the vertices [22. It is also one of the fundamental problems in graph theory and is applied to many areas in computer science. EDHC, also known as Hamiltonian decomposition, is an extension of the original HC problem. EDHC considers the problem of finding a family of HCs of a graph that are pairwise edge-disjoint. The HC and the EDHC problems are closely related to our work.

Section 2.1 and Section 2.2 provide a brief introduction to HC and EDHC. In Section 2.3. we present an interesting property of EDHC. Section 2.4 presents a heuristic algorithm for solving the EDHC problem.

\subsection{Hamiltonian Cycle}

In Section 1.2.3, we gave some examples of HC. Formally, for a graph $G=(V, E),|V|=n$, the $\mathrm{HC}$ problem is to find a cycle $C=\left(v_{1}, \ldots, v_{n}\right)$ such that $v_{i} \neq v_{j}$ for $i \neq j,\left(v_{i}, v_{i+1}\right) \in E$, and $\left(v_{1}, v_{n}\right) \in E$. The Hamiltonicity of a graph, which indicates whether or not a graph has a Hamilton cycle, is an important property often used in graph theory. 


\subsubsection{Basic Hamiltonian cycle theorems}

Now we will present some results on Hamiltons. Their proofs can be found in various graph theory text books [10] [48].

Lemma 1. If a graph contains a Hamilton cycle, then each vertex must have a degree greater or equal to 2.

Lemma 2. If a graph contains a Hamilton cycle, then all the edges connecting to the vertex which has exactly degree of 2 must be in any Hamilton cycle.

Lemma 3. If a vertex has 3 neighbors of degree 2, then that graph cannot contain a Hamilton cycle.

Lemma 4 (Dirac's Theorem). If $G=(V, E)$ is simple and $|V| \geq 3$ and minimum degree of the vertices is greater or equal to $|V| / 2(\delta(G) \geq|V| / 2)$, then $G$ contains at least one Hamilton cycle.

Lemma 5 (Ore's Theorem). If $G=(V, E)$ is a graph, where $|V| \geq 3$, and if for each pair of non-adjacent vertices $v$ and $w$, deg $(v)+\operatorname{deg}(v) \geq|V|$, then $G$ contains Hamilton cycle.

Lemma 6 (Bondy-Chvatal's Theorem). A graph is Hamiltonian if and only if its closure is Hamiltonian. (The closure of $G$ "is the graph obtained from $G$ by recursively joining pairs of nonadjacent vertices whose degree sum is at least $n$ until no such pair remains.")

\subsubsection{Hamiltonian cycle and random graph}

Random graph is a graph that can be generated by a random process. The theory of random graph lies at the intersection of graph theory and probability theory. In this section, we introduce two models of random graphs, $G_{n, p}$ and $G_{n, m}$. $G_{n, p}$ defines a graph with $n$ vertices and each of the $\left(\begin{array}{l}n \\ 2\end{array}\right)$ possible edge has an independent probability $p$ of being an edge in the random graph. So in the $G_{n, p}$ model the probability of having a graph of $n$ vertices and $q$ edges is $p^{q}(1-p)^{\left(\begin{array}{c}n \\ 2\end{array}\right)-q}$. The $G_{n, m}$ model, on the other hand, defines a graph with 
$n$ vertices and exactly $m$ edges and it is easy to see that the probability of a particular

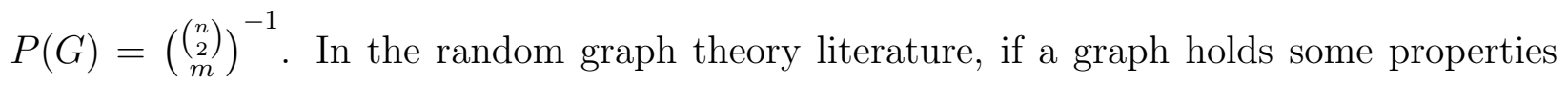
with probability $p=1-o(1)$ as $n \rightarrow \infty$, then the graph is said to hold the properties with high probability $(w h p)$.

In [36], Posa proved that given a random graph $G_{n, m}$ with $m=c n \log n$, it has a HC whp. Komlos and Szemeredi [33] extended this result as follows, for $m=\frac{n}{2}\left(\log n+\log \log n+c_{n}\right)$ :

$$
\lim _{x \rightarrow \infty} P\left(G_{n, m} \text { has a HC }\right)=\left\{\begin{aligned}
1 & \text { if } c_{n} \rightarrow \infty \\
e^{-e^{-c}} & \text { if } c_{n} \rightarrow c
\end{aligned}\right\}
$$

\subsubsection{Overview of Hamiltonian cycle algorithms}

It is known that the HC problem is NP-complete [10] and there is no effective (poly-time) algorithm to check for Hamiltonicity. So researches of $\mathrm{HC}$ algorithms look for effective heuristic and exhaustive search algorithms.

Heuristic algorithms usually run in linear or low-order polynomial time. They work by using some heuristic rules to guide their search of the possible solution space, but the drawback is that they may not return the optimal solution. Posa's algorithm [36] is a heuristic algorithm that attempts to extend the length of a partial path until a $\mathrm{HC}$ is formed. The algorithm starts with a single vertex and stops if a $\mathrm{HC}$ is found or no more extension to the cycle is possible. It does not permit backtracking. UHC [4] is similar to Posa's algorithm. One major difference is that the edges will be removed if they are selected to be used in partial path. DHC [4] for directed graph is very similar to UHC, but uses more complicated transformation for cases where it cannot extend the path from current endpoint. Bollobas, Fenner and Frieze [9] present another heuristic algorithm, HAM. The main idea is to extend a partial path until a HC is found. Several important techniques are introduced in HAM. HAM uses cycle extension when the partial path forms a cycle and uses breadth-first search to search for a possible extension to the current path. SparseHam [21] is a variation of HAM. They prove that the algorithms can find a HC - if it has one - in random graphs with constant 
average degree whp. SparseHam uses depth-first search for the path extension rather than breadth-first search in HAM. SpareHam has no limits on which path to search, and allows for paths to be repeatedly tried. Another difference between SpareHam and HAM is that SpareHam only considers extending the path from one of its endpoints. Broder, Frieze and Shamir [13] present an algorithm called HideHam; it finds HCs whp in graphs where a HC is hidden within a random graph. DB2 [14] is quite different from the other algorithms we discussed. DB2 considers $\mathrm{HC}$ as a special case of traveling salesmen problem in which the graph edges have weights. DB2 assign edges into different categories and prioritizs the edge selection process by these categories. Another innovation used by DB2 is to use vertex degree to prioritize vertex and edge selection. DB2A [14] can be used to find HC in directed graph. The main idea here is the conversion of a directed graph to an undirected graph and then execute DB2. Once the solution for the undirected graph is found, it simply converts the results back to the directed version. The LongPath algorithm [32] is very closely related to HC problem. The algorithm will report the maximum path (or cycle) found rather than return failure when no $\mathrm{HC}$ is found. In LongPath, three new techniques are introduced: crossover extension, chain extension and bypass extension. LinearHam [44] is a linear expected time algorithm. LinearHam contains three separate algorithms that are applied sequentially with increasing time requirements, but increasing probabilities of success.

Besides heuristic algorithms, backtracking can be used to solve the HC problem. Backtracking algorithms search the entire solution space, and pruning techniques (also known as branch cutting) are applied to prune the solution space. Backtracking algorithms usually guarantee the correctness of the result, but run in exponential time in the worst case. Kocay's Multipath [31] is a backtracking algorithm. It maintains several possible partial paths at the same time and later merges the partial paths into a complete HC. KTC algorithm [40] is another backtrack algorithm that employs many pruning rules to make the search process effective. 


\subsection{Edge-disjoint Hamiltonian Cycle (EDHC)}

As noted in Section 1.2.3, a set of HCs is called edge-disjoint if every edge in one HC is disjoint with every edge in the rest of the HCs in the set. Some of the results on HC can be easily extended to EDHC, as noted by many authors [10] [48].

Lemma 7. Given a graph $G$, let $\delta(G)$ be the minimum degree of vertex in $G$. Then the graph $G$ contain at most $\left\lfloor\frac{\delta(G)}{2}\right\rfloor E D H C$.

It should not surprise the reader that the EDHC problem is NP-complete, since the decision problem of showing the existence of even a single HC in graph is already known to be NP-complete. The NP-completeness of EDHC can be proved by restricting the edgedisjoint $\mathrm{HC}$ problem to a classic HC problem.

Theorem 1. Given a graph $G$ and an integer $h \geq 1$, the EDHC problem - whether $G$ contains a set of edge-disjoint Hamiltonian cycle whose cardinality is $h$ - is NP-complete.

Proof. We transform a generic HC problem to EDHC. Recall that HC is NP-complete [22]. Suppose graph $G(V, E)$ is the input to HC. Feed $G^{\prime}, G^{\prime}=G$, with $h=1$ to the corresponding EDHC instance. Clearly, if $G^{\prime}$ contains a set of EDHC whose cardinality is $1, G$ has a HC, else $G$ does not have a $\mathrm{HC}$.

Though we can conclude that finding the maximum number of EDHC is a NP-hard, it does not mean that we cannot find effective algorithms for some specific classes of graphs. Actually, most of the research in finding EDHC is focused on some specific classes of graphs.

Fully-connected graph is an undirected graph that has an edge between every pair of vertices. Formally, given a graph $G(V, E)$ if $|V|=n, 1 \leq i, j \leq n, \forall\left(v_{i}, v_{j}\right) \in E$, then the graph is called fully-connected graph. In [3], the author provides a method to generate a family of EDHC. For a fully connected graph with n vertex, the method will generate $\left\lfloor\frac{n-1}{2}\right\rfloor$ EDHCs. This method works by using a mathematical cycle permutation $\sigma[3]$. Note that 
$\sigma(H)^{k}$ means to apply the permutation $\sigma$ to the initial HC $H$, iteratively, $k$ times. A brief outline of their method is given below.

$$
H^{(k)}=\left\{\begin{aligned}
N_{1} N_{2} N_{3} N_{M} N_{4} N_{M-1} \ldots N_{1} & \text { if } k=1 \\
\sigma\left(H^{(1)}\right)^{k-1} & \text { if } k \neq 1
\end{aligned}\right\} .
$$

Since fully-connected graph is not always available or preferred in some cases, generating EDHC in other graphs has also been researched. Bae and Bose [6] give a method for finding EDHC in Torus and $k$-ary $n$-cubes by using Gray Codes. A polynomial time distributed algorithm for finding HCs in $k$-ary $n$-cubes and hypercubes is also given in [43].

\subsection{Order of Discovering EDHC}

A natural idea of finding EDHCs is to use an algorithm to find a HC first, and then delete all the edges in this HC. Repeat this process until there is no HC left in the graph. This method sounds reasonable, but is predicated on the notion that the order of finding HCs should not affect the number of EDHCs. Unfortunately, that may not the case. We will see that the number of EDHCs in a graph is dependent on the order in which the cycles are found.

Theorem 2. The order of finding edge-disjoint Hamilton cycles in random graph can affect the number of edge-disjoint Hamilton cycle one can possibly find.

Proof. We prove this by contradiction. Suppose Theorem 2 is false. It means that the order of finding $\mathrm{HC}$ should not matter. However, the example below clearly shows that the sequence of finding Hamilton cycle does affect the number of edge-disjoint Hamilton cycles in the graph.

It is easy to see that Figure 2.1 has two EDHCs. If the first $\mathrm{HC}$ we find is the solid line in Figure 2.1, there is no problem in finding another $\mathrm{HC}$ which is represented by the dotted line in Figure 2.1. But if the first Hamilton cycle is, instead, the one represented by the 


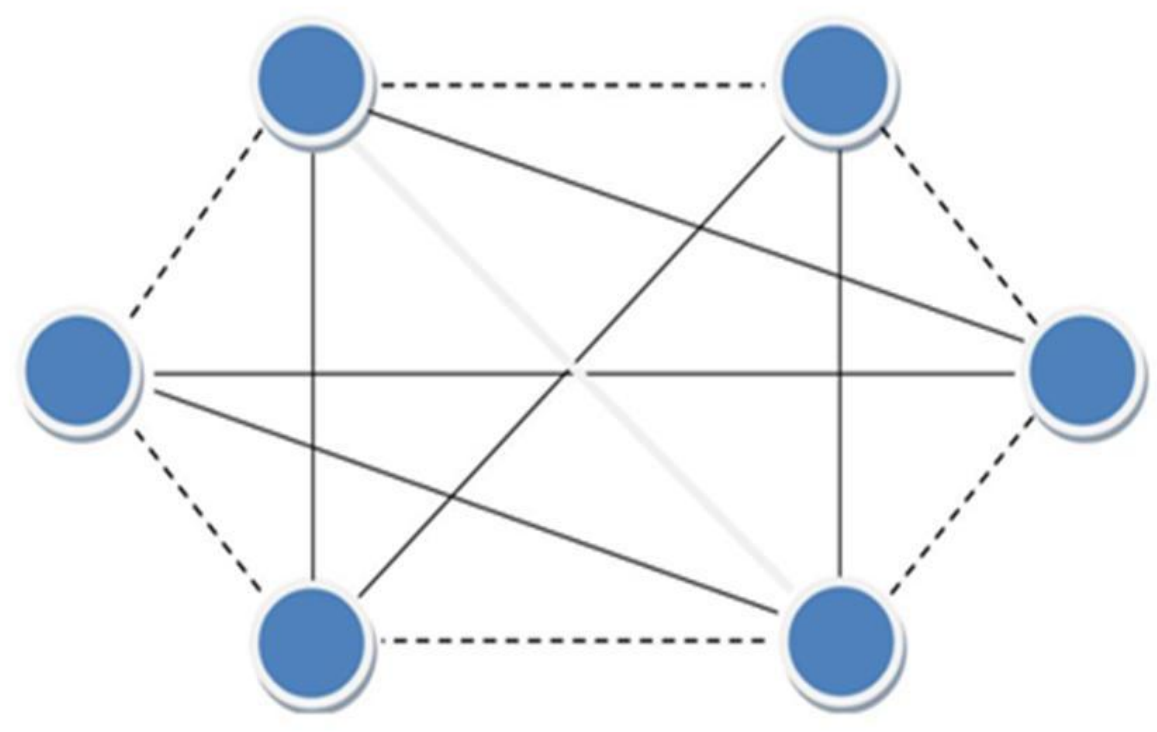

Figure 2.1: Order of Finding EDHC

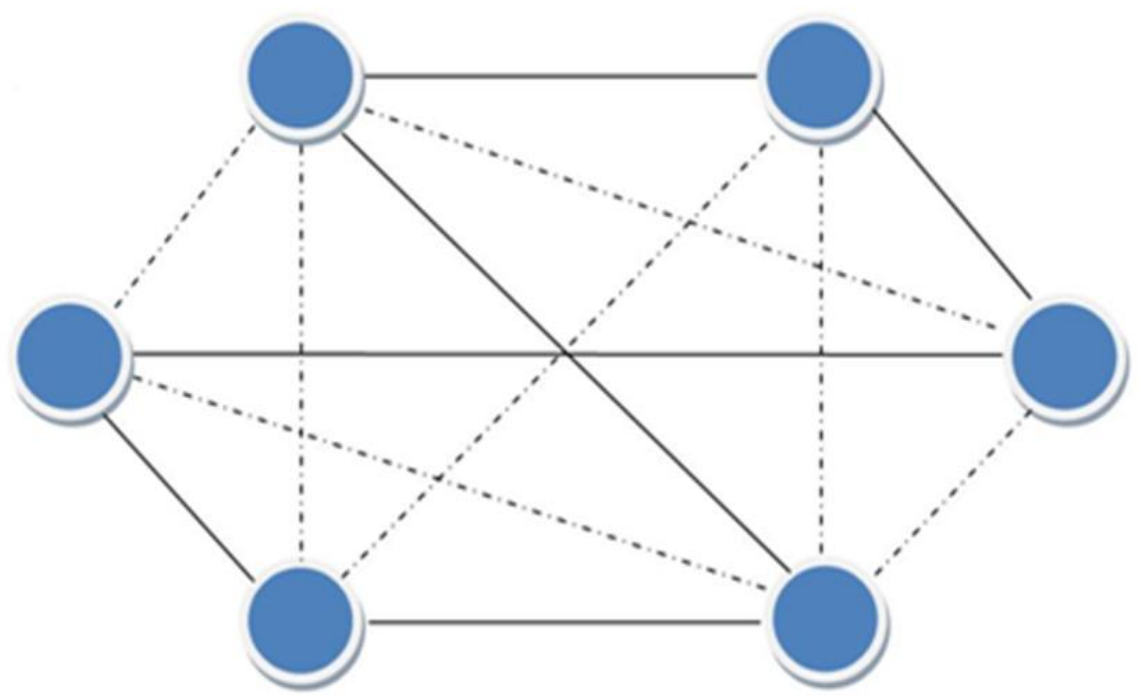

Figure 2.2: Another Order of Finding EDHC

solid line in Figure 2.2, we can find no more HC, with the remaining edges in the graph as represented by the dotted lines in Figure 2.2.

In order to explain this "sequence-dependence" phenomenon, we realize that it is important to identify the "kernel" of an EDHC problem - the part of the problem which makes 
the problem hard to solve. Since classic HC problem is already NP-complete, we want to know that can we solve the EDHC problem if we have an "oracle machine" which can solve any classic $\mathrm{HC}$ problem in a constant time $\mathrm{O}(1)$. Here we assume that our oracle machine can list all the HCs in a given graph within a constant time! Note, the oracle machine we assume here is an abstract machine which means it does not actually exist or at least not discovered yet. With the existence of the oracle machine, the EDHC problem becomes this: given a graph and a list of $\mathrm{HC}$ in the graph, what is the maximum number of edge-disjoint cycle in the list?

Now let us consider this problem: given an undirected graph $G(V, E)$, a cycle set $C$ and a positive integer $k$, does it have a cycle set $S$ which makes $|S| \geq k$ where $S \subseteq C, \forall S_{i}, S_{j} \in S$, $S_{i}$ is edge disjoint to $S_{j}(i \neq j)$ ? We name this problem as Restricted Edge-Disjoint Cycle (REDC); any cycle here is restricted to a member of $C$. Note, though it looks very similar to edge-disjoint cycle problem (EDC) [22], the problem here is not quite the same.

We actually show that the problem of finding the largest edge-disjoint cycle set - from a given graph and a set of cycles - is NP-complete. We prove this by transforming the maximum clique problem (MC) to REDC in polynomial time. An introduction to $\mathrm{MC}$ problem is given in [22]. For MC, given an undirected graph $G$ and a positive integer $k$, we ask if graph $G$ has a fully-connected subgraph $S G(V, E)$ with the constraint $|S G(V)| \geq k$.

Theorem 3. REDC is NP-complete

Proof. We transform MC to REDC. Suppose $G(V, E)$ is an instance of the MC problem. Convert $G$ to another graph $R G(V, E)$ and a set of cycles $R C$. Both $R G$ and $R C$ can be considered as input to REDC. For any $G(V, E)$ with $|G(V)|=n$, we construct $R G(V, E)$, such that $|R G(V)|=n^{2}+n, v_{i, j} \in R G(V), 1 \leq i \leq n, 0 \leq j \leq n$, cycle set $R C$ with $|R C|=n$ for $1 \leq i \leq n$, and initialize $R C_{i}=\left(v_{i, 0}, v_{i, 1}, v_{i, 2}, \ldots, v_{i, n}, v_{i, 0}\right)$. Now we modify $R C$ a bit, so the result of REDC can be used as a solution to MC with input $G(V, E)$. For each $V_{i} \in G(V)$ if $\left(v_{i}, v_{j}\right) \notin G(E)$ and $i \leq j$, insert $\left(v_{j, i-1}, v_{j, i}\right)$ into $R C_{i}$ after the vertex $v_{i, i-1}$ and add edge as needed. It can be seen that the complexity of this transform is $O\left(n^{2}\right)$. 
The purpose of the transform is to make $R C_{i}$ edge-joint to $R C_{j}$ if $\left(v_{i}, v_{j}\right) \notin G(E)$. And, for any $\left(v_{i}, v_{j}\right) \in G(E), R C_{i}$ and $R C_{j}$ are always edge-disjoint to each other. It is clear that if we have a set of edge-disjoint cycle $S R C, S R C \subseteq R C$, in $R G$ and $|S R C|=k$ we have a fully connected subgraph $S G \subseteq G$ with $|S G(V)|=k$.

Figure 2.3 and Figure 2.4, show how to transform an instance of an MC problem with input $G(V, E)$ to the REDC problem with input $R G(V, E)$ and a set of cycles $R C=$ $\left\{R C_{1}, R C_{2}, \ldots, R C_{|V(G)|}\right\}$. Figure 2.4 has a cycle corresponding to each node of the Figure 2.3. Node $i$ of Figure 2.3 is represented by $R C_{i}$ in Figure 2.4. For example, node 2 in Figure 2.3 is represented by $R C_{2}$ (the black cycle) in Figure 2.4. If two nodes have an edge between them in Figure 2.3, the two corresponding cycles of Figure 2.4 are edge-disjoint. For example, $R C_{1}$ and $R C_{2}$, are edge-disjoint, since node 1 and 2 have an edge between them in Figure 2.3. It can be seen that only $R C_{1}, R C_{2}$ and $R C_{3}$ are edge-disjoint to each other, and this indicates that in the original $\mathrm{MC}$ problem vertex 1, 2 and 3 form a fully connected subgraph or Figure 2.3 has a clique of size 3.

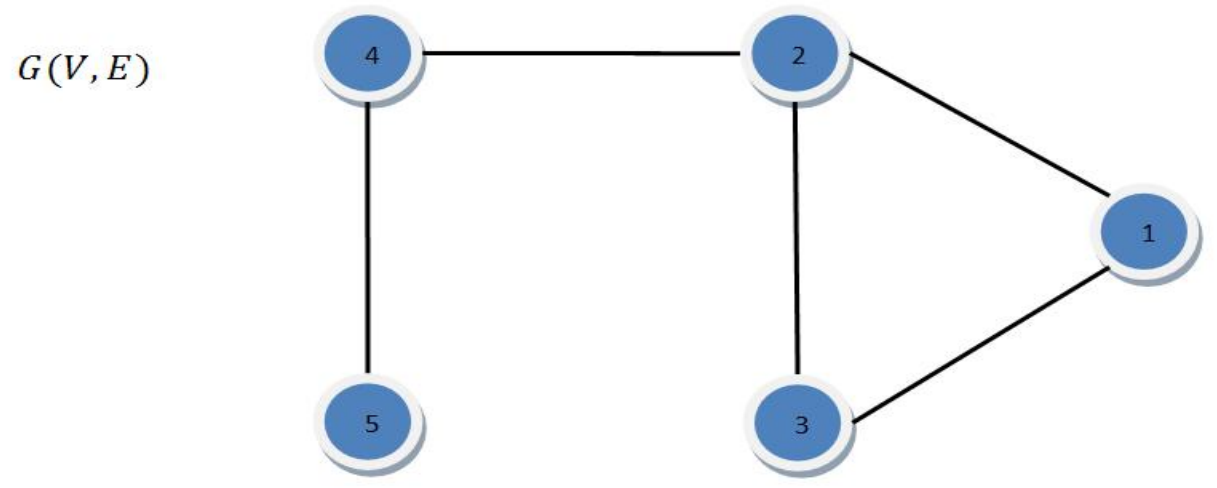

Figure 2.3: Maximum Clique of Size 3

Based on the NP-completeness of REDC, we conjecture that EDHC is an NP-complete problem under the assumption of an oracle machine to discover HC. This result helps explain why the sequence of finding HCs will affect the maximum number of EDHCs we can find. If the sequence does not matter, it means - with an oracle machine solving $\mathrm{HC}$ problem - we 


$$
\begin{aligned}
& R C_{1}=\left(v_{1,0}, v_{4,0}, v_{4,1}, v_{5,0}, v_{5,1}, v_{1,1}, v_{1,2}, v_{1,3}, v_{1,4}, v_{1,5}, v_{1,0}\right), \\
& R C_{2}=\left(v_{2,0}, v_{2,1}, v_{5,1}, v_{5,2}, v_{2,3}, v_{2,4}, v_{2,5}, v_{2,0}\right), \\
& R C_{3}=\left(v_{3,0}, v_{3,1}, v_{3,2}, v_{4,2}, v_{4,3}, v_{5,2}, v_{5,3}, v_{3,3}, v_{3,4}, v_{3,5}, v_{3,0}\right), \\
& R C_{4}=\left(v_{4,0}, v_{4,1}, v_{4,2}, v_{4,3}, v_{4,4}, v_{4,5}, v_{4,0}\right), \\
& R C_{5}=\left(v_{5,0}, v_{5,1}, v_{5,2}, v_{5,3}, v_{5,4}, v_{5,5}, v_{5,0}\right)
\end{aligned}
$$

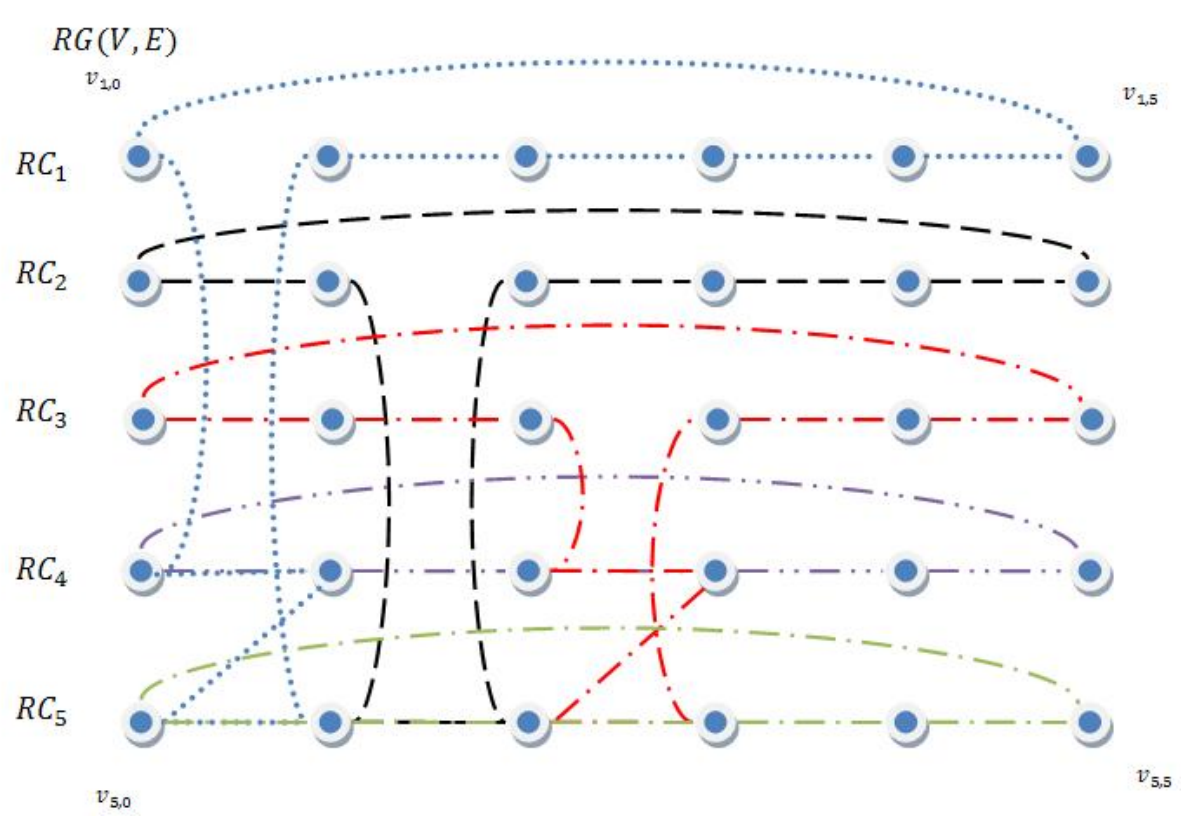

Figure 2.4: Transform MC to REDC

have a linear time algorithm to solve EDHC. Obviously, this is in conflict with the Theorem 3 where we showed REDC is NP-complete.

Note that in the above paragraph, we conjectured that EDHC is NP-complete even with an oracle machine solving the classic HC problem. This is because given an oracle machine, the EDHC problem becomes this: given a graph, a positive integer $k$ and a set of all the HCs in this graph, find whether there exists $k$ edge-disjoint cycles in that set. This problem is not identical to the REDC problem, since it is a special case of REDC problem. We note that it is possible that some special case of an NP-complete problem may not be NP-complete. 


\subsection{Heuristic Algorithms for EDHC}

In the previous section, we showed that the problem of finding the maximum number of EDHC in an arbitrary graph is NP-hard, and the decision problem of whether a given graph has a given number of EDHC is also NP-complete. Just like many other NP-complete and NP-hard problems, we do not have any efficient (polynomial time) algorithm for the EDHC problem. Heuristic algorithms, which work by using some heuristic rules to guide their search of the solution space, are powerful tools to solve NP-hard problems. However, they may converge to an approximate or suboptimal solution. In this section, we will first present a connection between the $\mathrm{EDHC}$ problem and $\mathrm{MC}$ problem. In the later part of this section, we will provide a heuristic algorithm for EDHC problem.

$\mathrm{MC}$ is one of the fundamental NP-complete problems [22] and is well researched. The purpose of finding a connection between EDHC and $\mathrm{MC}$ is to apply some of the results from $\mathrm{MC}$ to the EDHC. We present a procedure to transform the EDHC problem to MC. Note, we still assume that we have an oracle machine that lists all the HCs in a given graph. The transform works as follows: take a graph $G(V, E)$ as an input to the EDHC problem; then use the oracle machine to obtain a set $H$ which contains all the $\mathrm{HC}$ in the given graph; finally, construct another graph $G^{\prime}(V, E)$ in which each vertex in $G^{\prime}$ has a corresponding $\mathrm{HC}$ in the set $H$, and if two elements in $H$ is edge-disjoint then there is an edge between the two corresponding vertices in $G^{\prime}$ and vice versa. Now, solving the MC problem in $G^{\prime}$ gives us the results for the original EDHC problem.

Before we go any further, we present a short survey of MC algorithms. Reactive local search (RLS) [7] is a method derived from reactive Tabu search [8]. RLS adapts tabu tenure parameter automatically during the search process, and uses a dynamic restart strategy to provide long-term diversification. In [15], the authors give a deterministic iterative greedy construction algorithm called QUALEX-MS that uses vertex weights derived from a nonlinear programming formulation of MC. Another greedy construction algorithm, Deep Adaptive Greedy Search (DAGS), is given in [23]. The $k$-opt algorithm [29] is based on a 
simple variable depth search procedure that uses elementary search step in which a vertex is added to or removed from the current clique. In [41] a recent ant colony optimization algorithm for MC problem called Edge-AC+LS is presented. Finally, a stochastic local search called Dynamic Local Search (DLS-MC) is presented in [37]. According to these authors, the DLS-MC outperforms other state-of-the-art MC search algorithms in many instances.

In our work, we will take DLS-MC [37] as a basis to construct a dynamic local search algorithm to solve EDHC. We explain DLS-MC in this section and discuss our dynamic local search algorithm in Section 2.4.1.

DLS-MC algorithm works as follows: start with a random initial vertex from the given graph as a current clique $C$. Then the search alternates between an iterative improvement phase and a plateau search phase. In the iterative improvement (or expand) phase, suitable vertices (connect to each element in current clique) are repeatedly added to current clique. Let $N I(C)$ the set of all these vertices. The expand phase terminates when $N I(C)$ is empty. In the plateau search phase, one vertex of current clique $C$ is swapped with a vertex currently not in $C$. Let $N L(C)$ the set of all the vertices that connect to $|C|-1$ vertices in $C$. The plateau search terminates when $N L(C)$ is empty. Another termination condition [29] is that when there is no overlap between current clique $C$, and $C^{\prime}$ - the current clique at the start of the plateau search. Following the plateau search, a numerical value is added to each vertex as vertex penalties. And penalty delay - a parameter of the algorithm - is designed to prevent penalty values from becoming too large and allows DLS-MC to even 'forget' penalty values over time. The algorithm repeats the above steps - expand phase and plateau phase - until large enough clique is found or the step limitation is reached. Algorithm details and empirical results can be found in [37].

As noted earlier, we can transform the EDHC problem to $\mathrm{MC}$, and there are many algorithms for solving MC. The only barrier left is the existence of the oracle machine in our transform at the beginning of this section. Our solution is to replace the oracle machine with some heuristic algorithms that we discussed in Section 2.1.3. We will not try to use 
heuristics to generate all the HCs - an impossible task. Instead, we will dynamically construct the graph for the DLS-MC program. In our algorithm, we will consider a heuristic algorithm for finding $\mathrm{HC}$ as a component since it is very hard to determine what the best heuristic $\mathrm{HC}$ algorithm is. In practice, one can choose a heuristic $\mathrm{HC}$ algorithm based on his/her needs because algorithms that need more accuracy may take up more computing time. In our work, all heuristic HC algorithms take two parameters as input, a graph and a random number. We know that most of the heuristic $\mathrm{HC}$ algorithms start with picking an edge in the graph. The purpose of the random number is to give the algorithm an opportunity to return a different $\mathrm{HC}$ each time. All algorithms return a $\mathrm{HC}$ if it finds a new $\mathrm{HC}$ else report 'failure.' Note in our work, before the algorithm returns any HC, it will check whether it is a new HC (never found before) or not. In order to implement this, a global value $H$ is used to store all the $\mathrm{HC}$ we have found so far and their edge-disjoint relationships. For notational convenience, we use the term $H H C(G, r)$ to mean any $\mathrm{HC}$ algorithm with parameters $G$ and $r$.

\subsubsection{Heuristic dynamic local search - EDHC}

Based on the above considerations, we introduce a new heuristic algorithm (Figure 2.5-2.7) for edge-disjoint HC, Heuristic Dynamic Local Search-EDHC (HDLS-EDHC). An outline of the algorithm is given in Figure 2.5. For the given graph $G(V, C)$, we start with finding a set of EDHC as current solution $C H$ by expandSearch (Figure 2.6). Then, we execute expandSearch and plateauSearch (Figure 2.7) alternatively. The process repeats until enough EDHC are found or step limit is reached.

In expandSearch (Figure 2.6), we construct a graph $G^{\prime}$ by removing all the HCs in $C H$ from $G$, and feed $H H C$ with $G^{\prime}$. It is obvious that any new $\mathrm{HC}$ found by $H H C\left(G^{\prime}, r\right)$ can be used to expend the size of $C H$. If $H H C\left(G^{\prime}, r\right)$ returns failure (which means no new $\mathrm{HC}$ is found), we will try to look at possible cycles in set $H$ (which contains all the HC we already found). Set $N I(H, C H)$ denotes the set of all the cycles in $H$ which can be added to $C H$. If 
$|N I(H, C H)|>1$, choose the one with least penalty. Repeat this process until $H H C\left(G^{\prime}, r\right)$ returns failed and $N I(H, C H) \neq \emptyset$. If no cycle is added during the expandSearch process, the expandSearch returns 'failure.' Otherwise, expandSearch returns both the $C H$ and hlast which is the last $\mathrm{HC}$ added to $C H$.

In plateauSearch (Figure 2.7), we construct a set of graph $G^{\prime \prime}$ by combining any $\mathrm{HC}$ in $\mathrm{CH}$ with the remaining graph $G^{\prime}$ (after removing all the $\mathrm{HC}$ in $\mathrm{CH}$ from $\mathrm{G}$ ). So we have $|C H|$ possible $G^{\prime \prime}$. Feed HHC with each $G^{\prime \prime}$; if any $H H C\left(G^{\prime \prime}, r\right)$ returns a new HC, then replace the new $\mathrm{HC}$ with the one used to construct $G^{\prime \prime}$. Let $N L(H, C H)$ denotes the set of all the cycle in $H$ which is edge-disjoint to all the cycles in $\mathrm{CH}$ except for one cycle. If all $H H C\left(G^{\prime \prime}, r\right)$ return nothing, then try to find a cycle in set $N L(H, C H)$ with least penalty to replace a cycle in $C H$ and maintain $C H$ pairwise edge-disjoint at the same time. Continue doing this until it is possible to do the expandSearch again $((H, C H) \neq \emptyset$ or $H H C\left(G^{\prime}, r\right) \neq$ failure) or $C H$ is completely different from $C H^{\prime}$ where $C H^{\prime}$ was saved before initiating plateauSearch.

At the end of plateauSearch, the cycle penalties are updated by incrementing the penalty values of all cycles in $C H$ by 1 . If no more move is possible after search process, the $C H$ will be reset to the last cycle added to the $C H$.

In sum, following the computation of the algorithm (Figure 2.5), we use the best solution found in the search process as the result, given by $C H$ - the set of EDHC in $G$. 


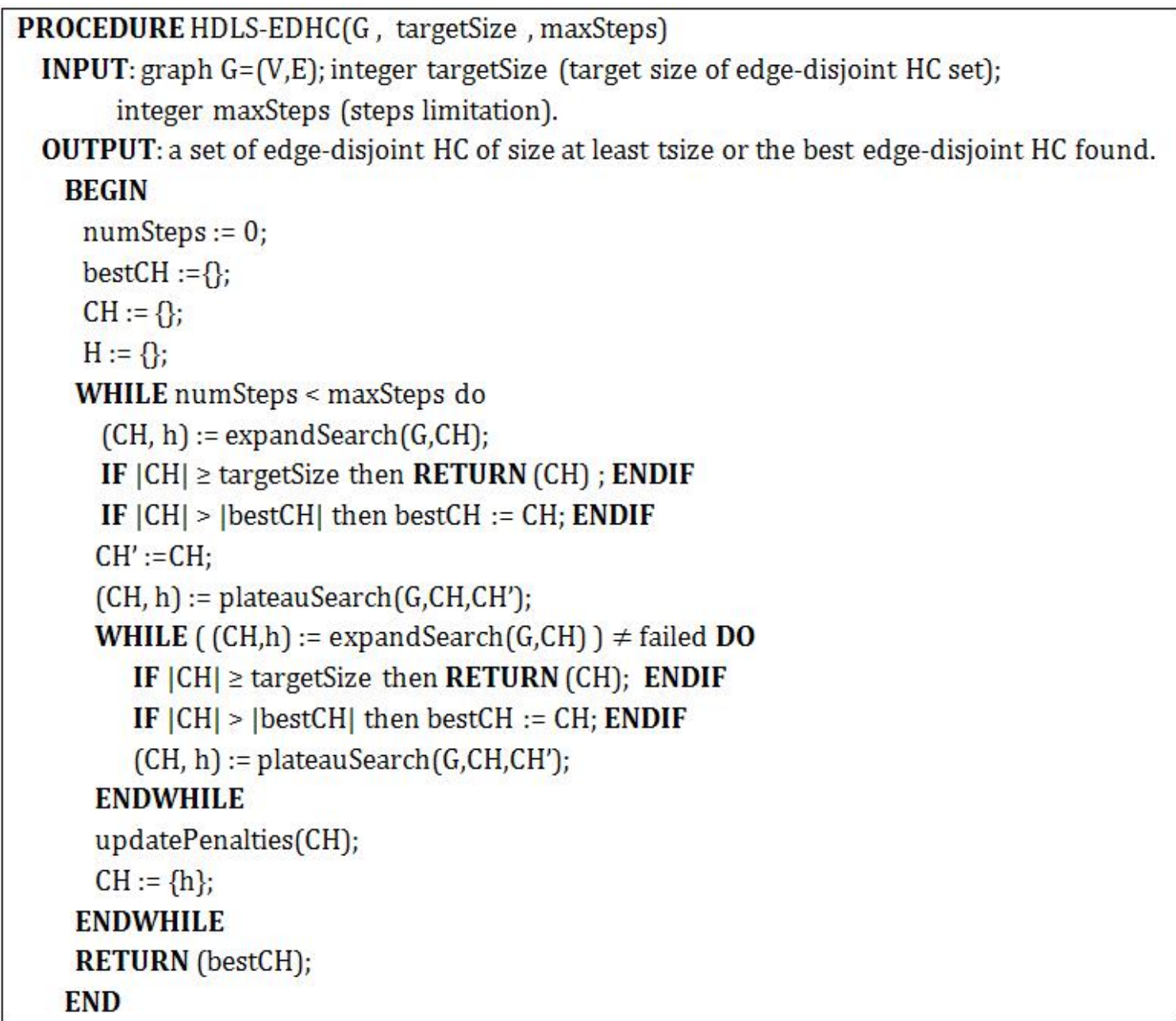

Figure 2.5: HDLS-EDHC 


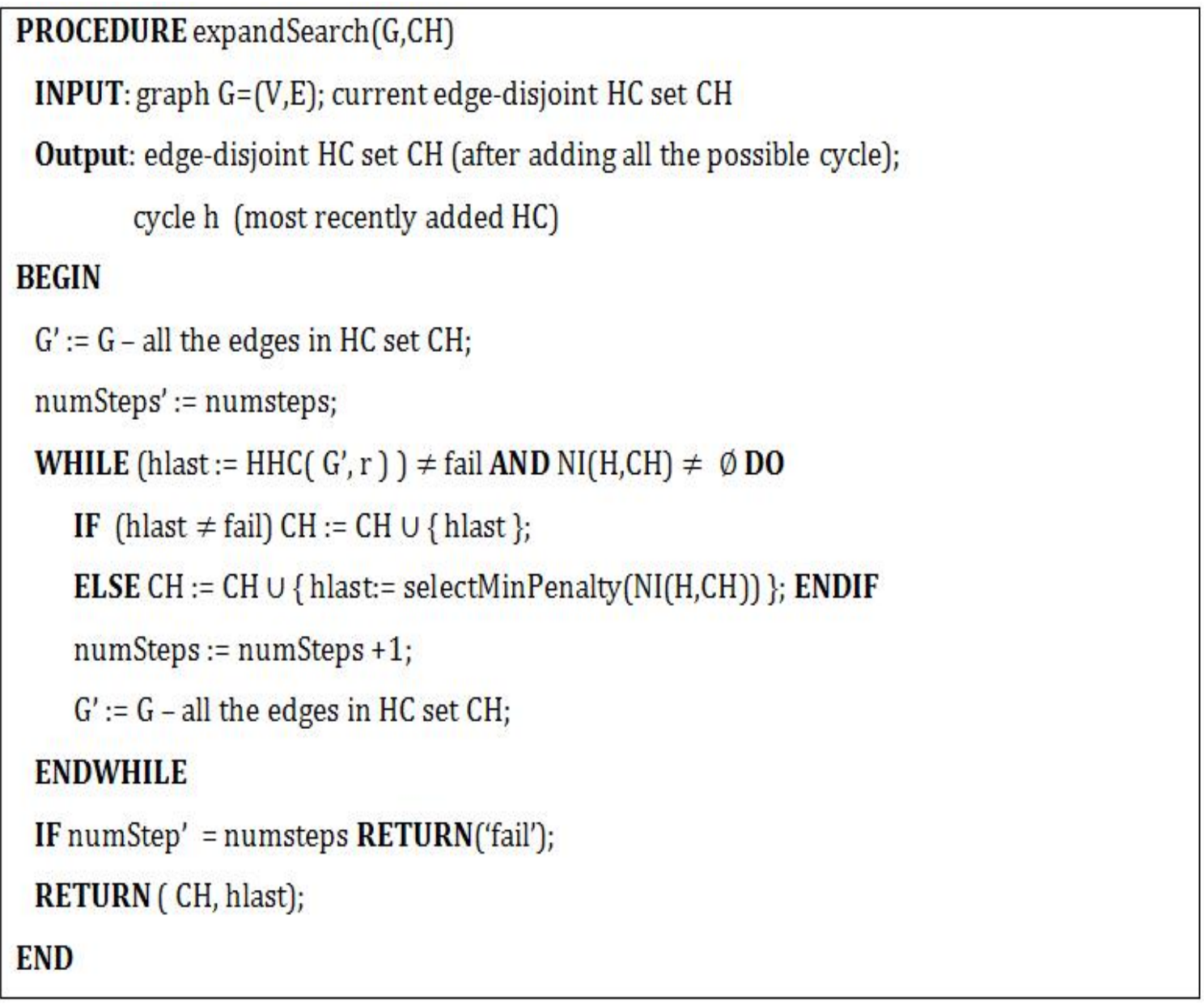

Figure 2.6: HDLS-EDHC - expandSearch 


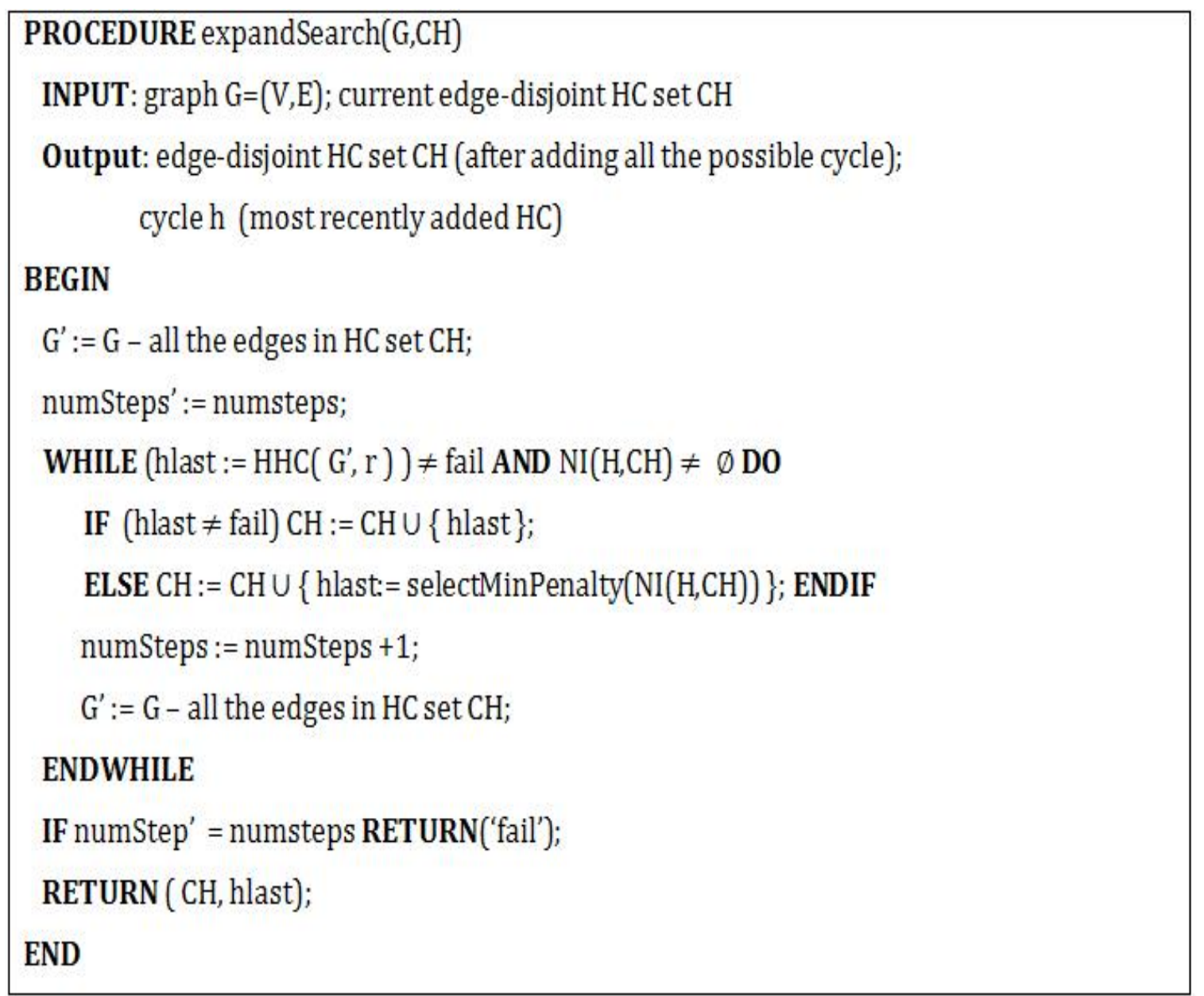

Figure 2.7: HDLS-EDHC - plateauSearch 


\section{CHAPTER 3}

\section{TRUST ENABLED SECURE}

\section{MULTIPARTY COMPUTATION}

In this chapter we focus on secure multiparty computation (SMC), especially those that are based on the application of HC. We first give an introduction to HC based SMC. In Section 3.2 , we present an example which provides the motivation for our attempts to improve the security of SMC. Then, we extend it by providing a generalized trust model in Section 3.3 . In Section 3.4, we present a greedy algorithm that achieves good values for the metric defined in the trust model of Section 3.3 .

\subsection{Hamiltonian Cycle Based SMC}

Many SMC methods have been developed to solve a number of derivatives of SMC problems [18]. Some of these methods are based on the application of HC. Here, we call an SMC as HC-based, also known as HSMC, if the computation of the algorithm relies on one or more HCs to obtain the global result. Figure 3.1 (like Figure 1.1 of Chapter 1) shows an example of HSMC. Assume the presence of a $\mathrm{HC}$ as shown in Figure 3.1. The algorithm initiates a SS computation starting at the source station $p_{1}$ whose private value is $x_{1}=8 . p_{1}$ uses a random $r_{x}, r_{x}=5$, to mask its private value [39]. Then $p_{1}$ sends $x_{1}+r_{x}=13$ to the next 
station. $p_{2}$ receives $x_{1}+r_{x}=13$ and adds its local value, $x_{2}$, to the sum before sending it to the next station. At the end, start node, $p_{1}$, receives $r_{x}+\sum_{i=1}^{5} x_{i}$. Since $p_{1}$ selected $r_{x}=5$, the global sum can be easily calculated. A similar algorithm can also be used to compute secure set union and secure set intersection [18].

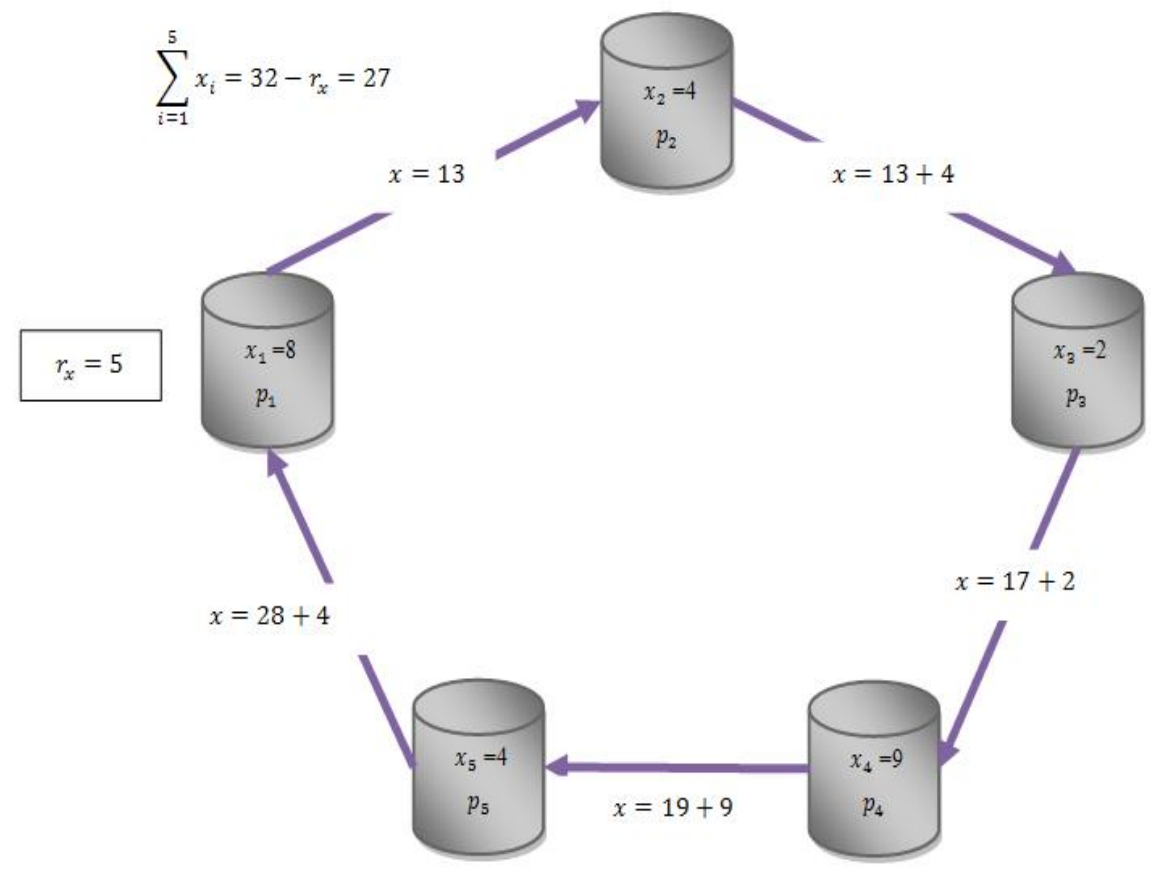

Figure 3.1: Secure Sum Computation - One Cycle

One of the disadvantages of the method presented in Figure 3.1 is that it can be vulnerable to collusion attack. For example, in Figure 3.1, $x_{3}$ can be easily found if $p_{2}$ and $p_{4}$ collude. In order to make HSMC more secure to collusion attack, cycle-partitioned SMC algorithm is developed in [45] and modified in [46]. The later algorithm, known as cycle partitioned secure sum computation (CPSS), improved the original algorithm by using several HCs. An example of CPSS is given in Figure 3.2. In Figure 3.2, $p_{3}$ is connected to 4 other participants which means that at least 4 other participants have to collude - or join together - in order to discover $p_{3}{ }^{\prime} s$ contribution to the global sum. Actually, if we use n HCs, one can prove that CPSS is $2 n-1$ collusion resistant, i.e. at least $2 n$ participants have to collude to find out 


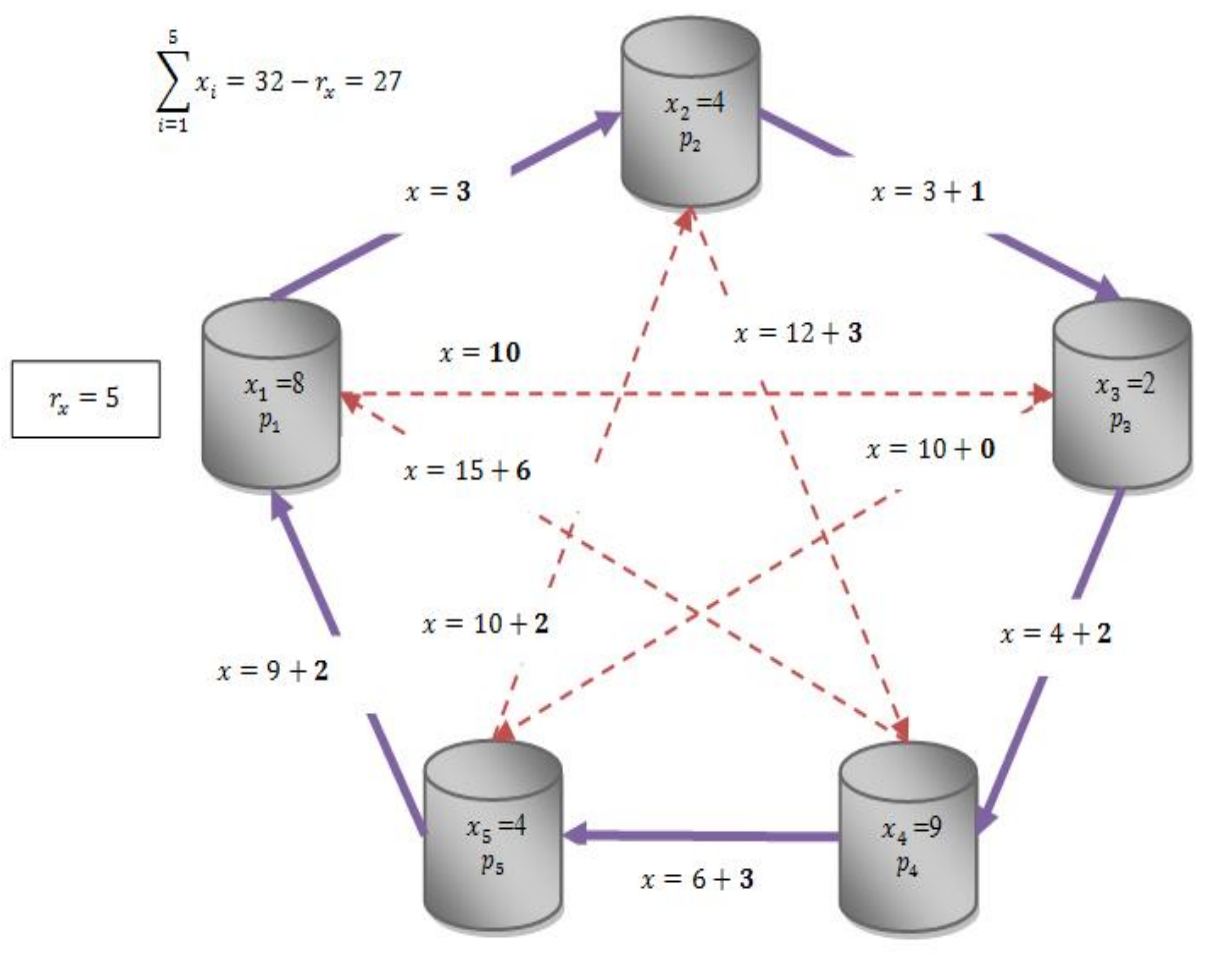

Figure 3.2: Secure Sum Computation - Two Cycles

someone else's contribution. A mathematical proof can be found in [39].

Note that the HCs used in Figure 3.2 are edge-disjoint. However, the number of EDHC in a given graph is usually very limited. The limited number of EDHCs in the graph constrains the application of CPSS algorithms. So one interesting question is how to defend against collusion attack without using too many EDHCs.

\subsection{Notion of Trust}

In the previous section, we discussed HSMC and the reliance on EDHCs to prevent collusion attack. In this section we first give an example to explain how we can improve the security of the algorithms without using additional EDHC. Then, we propose a mechanism which we believe will help us improve the overall security of HSMC. 


\subsubsection{Defend against collusion attack}

In all of the algorithms presented in the literature, each participant is no more or no less trustworthy than other participants. However, in the real world, participants are all not homogeneous, and we believe this information can be used to improve the collusion resisting ability of HSMC. We first explain our idea with an example. Consider mining data in a network of 7 supermarkets, A, B, C, .., G. Of these 7 supermarkets, suppose company W owns first 4; company M owns next two, and G is locally-owned. Clearly, two supermarkets are likely to trust each other more if they are owned by the same parent company.

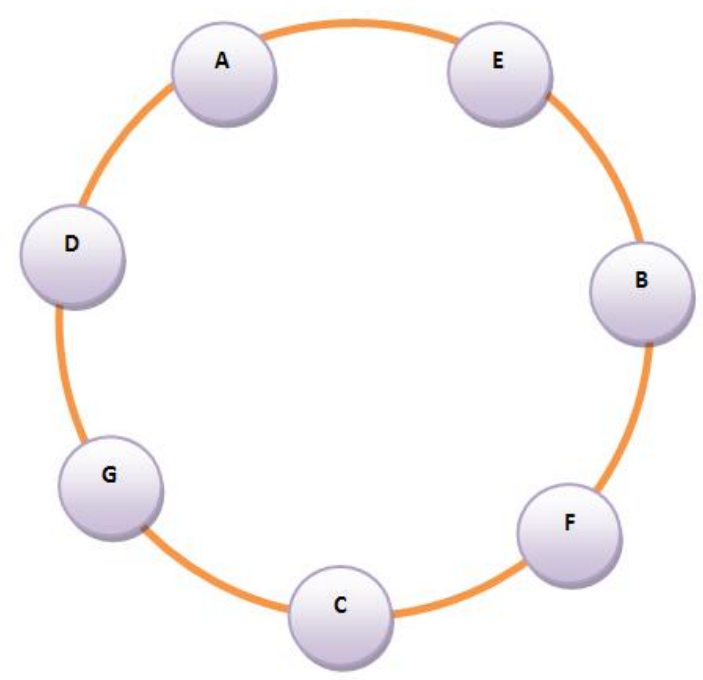

Figure 3.3: Configuration of SMC

Figure 3.3 shows a HC visit of these 7 supermarkets for use in HSMC. However, HSMC with this cycle is pretty vulnerable. For example, since E's left and right neighbors (A and B) are both owned by a same parent company (W), it is hard for E to keep its input secret if A and B collude. Unfortunately, such collusion is likely to happen, because A and B trust each other (more). Actually, the same problem may happen to any participant whose neighbors are not trustworthy. Suppose, we rearrange the HC of Figure 3.3 to obtain Figure 3.4 . supermarkets that belong to the same parent company are located next to each other. Given 


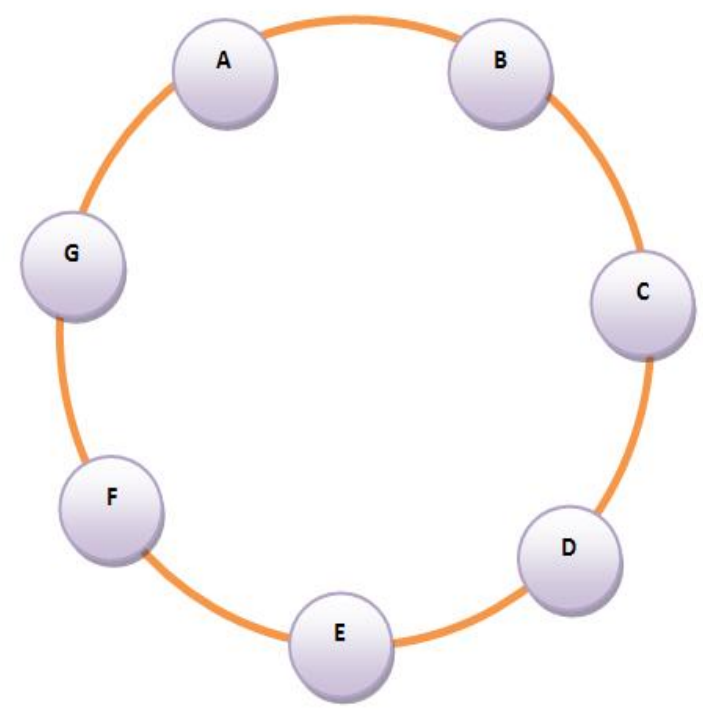

Figure 3.4: An Improved Configuration of SMC

the trust relationship between $\mathrm{A}, \mathrm{B}$ and $\mathrm{C}$, collusion by $\mathrm{A}$ and $\mathrm{C}$ to discover the private value of B is less likely. The purpose of this reconfigured cycle is to make participants surrounded by other participants that are more trustworthy. Intuitively, configuration Figure 3.4 is safer than that of Figure 3.3 .

\subsection{A Model for Trust Enabled HSMC (TE-HSMC)}

In the previous section, we compared two different configurations of HSMC and noted that the network can be made more secure by rearranging the $\mathrm{HC}$ for HSMC. The motivation behind the approach was the notion of trust. In this section, we formulate a generalized model, trust enabled HSMC (TE-HSMC), to define trust levels and their impact on HSMC. In our model, each participant gives every other participant a numeric trust level in the range 0 to $100 \%$. For example, A trusts B $90 \%$ (denoted as $T_{A, B}$ ) meaning that A believes there is only a $10 \%\left(1-T_{A, B}\right)$ chance that $\mathrm{B}$ will leak information to the others, about $\mathrm{A}$, during the SMC process. Table 3.1 provides an example of a trust level matrix, where each participant assigns a trust level to every other participant. 


\begin{tabular}{|c|r|r|r|r|r|r|r|}
\hline$T_{i, j}$ & $\mathbf{A}$ & $\mathbf{B}$ & $\mathbf{C}$ & $\mathbf{D}$ & $\mathbf{E}$ & $\mathbf{F}$ & $\mathbf{G}$ \\
\hline $\mathbf{A}$ & $\mathbf{1}$ & $90 \%$ & $95 \%$ & $69 \%$ & $45 \%$ & $30 \%$ & $5 \%$ \\
\hline $\mathbf{B}$ & $94 \%$ & $\mathbf{1}$ & $93 \%$ & $82 \%$ & $50 \%$ & $20 \%$ & $70 \%$ \\
\hline $\mathbf{C}$ & $85 \%$ & $92 \%$ & $\mathbf{1}$ & $78 \%$ & $25 \%$ & $12 \%$ & $40 \%$ \\
\hline $\mathbf{D}$ & $75 \%$ & $90 \%$ & $88 \%$ & $\mathbf{1}$ & $15 \%$ & $40 \%$ & $28 \%$ \\
\hline $\mathbf{E}$ & $20 \%$ & $38 \%$ & $10 \%$ & $18 \%$ & $\mathbf{1}$ & $90 \%$ & $38 \%$ \\
\hline $\mathbf{F}$ & $29 \%$ & $31 \%$ & $43 \%$ & $21 \%$ & $99 \%$ & $\mathbf{1}$ & $29 \%$ \\
\hline $\mathbf{G}$ & $14 \%$ & $13 \%$ & $31 \%$ & $27 \%$ & $20 \%$ & $20 \%$ & $\mathbf{1}$ \\
\hline
\end{tabular}

Table 3.1: A Trust Matrix

With the trust matrix, we want to quantify the 'Safety Level,' $S_{i}$, of participant $p_{i}$ in the network. From the previous section, we know that the safety of a participant depends on the reliability of its neighbors. For each participant $p_{i}$, the only way for the others to find out $p_{i}^{\prime} s$ contribution is for all the neighbors of $p_{i}$ to collude in order to disclose $p_{i}^{\prime} s$ information to a third party. Based on this consideration, Equation (3.1) below defines the safety level, $S_{i}$, of $p_{i}$ where $N\left(p_{i}\right)$ is the number of direct neighbors of node $p_{i}$ in HSMC. Actually, $S_{i}$ is the likelihood that $p_{i}{ }^{\prime} s$ contribution will be secure. i.e. the chance that at least one of its neighbors does not disclose information against $p_{i}$, making collusion by all of its neighbors unlikely - at least not a certainty - from a probabilistic viewpoint.

$$
S_{i}=1-\prod_{p_{j} \in N\left(p_{i}\right)}\left(1-T_{i, j}\right)
$$

Figure 3.5 gives an example of computing $S_{A}$ of node A using the data from Table 3.1. In Figure 3.5, A has two direct neighbors, F and B. $S_{A}$ can be computed using Equation (3.1), and is seen to be 0.93 .

Figure 3.6 is a more detailed example. Here, we see that A - between the two HCs - has 4 direct neighbors, i.e. A's contribution to the SMC can be revealed if these 4 nodes collude. With the trust level given by Table 3.1. Equation 3.1 is used to compute the safety level, $S_{A}$, of node A. From Figure 3.6, $S_{A}=94.1 \%$. As noted earlier, $S_{A}$ measures the likelihood that node A's contribution will be secure, i.e. the chance that at least one of its neighbors 
declines collusion!

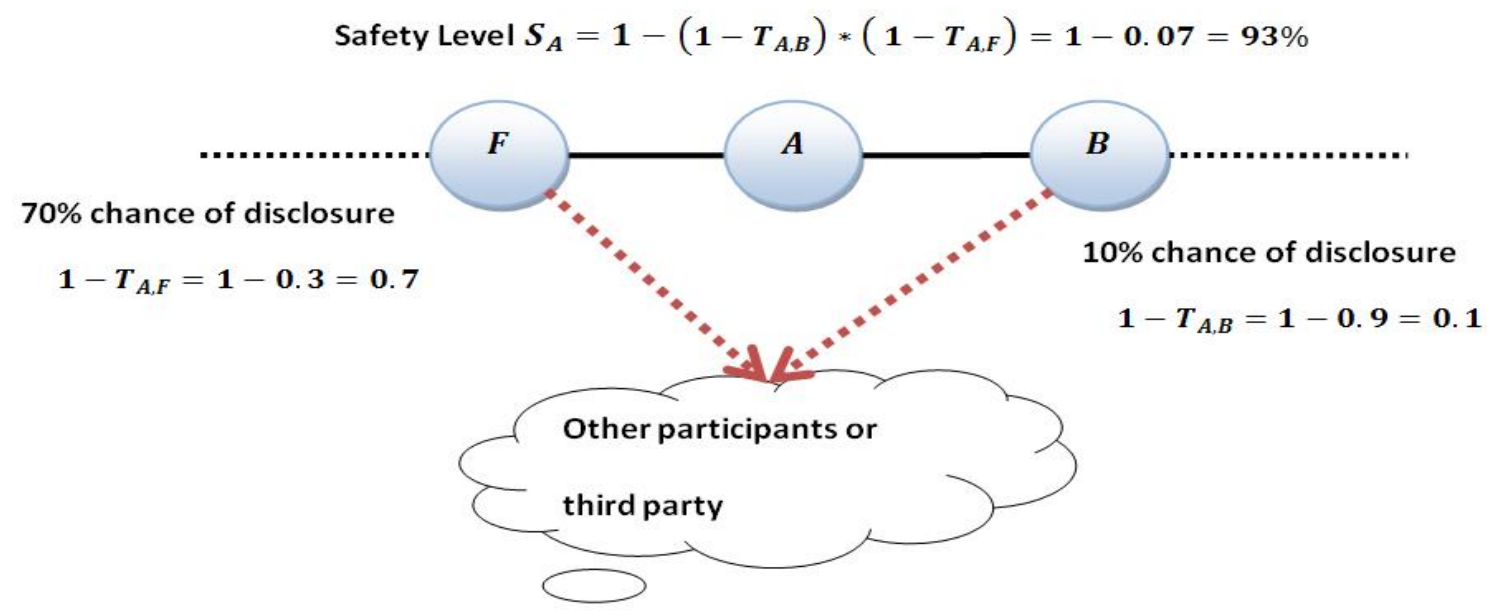

Figure 3.5: Safety Level of Node A

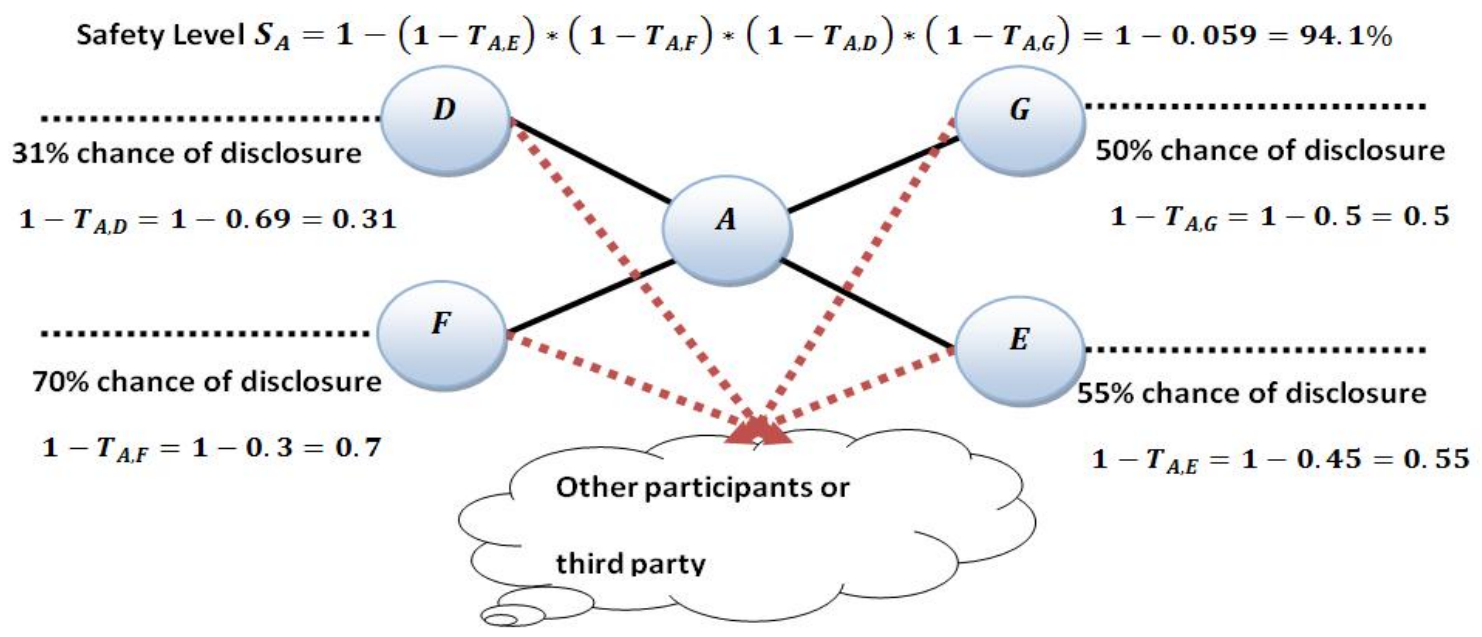

Figure 3.6: Safety Level with CPSS

Now, using the trust level matrix and the safety level defined above, we can convert the TE-HSMC problem into an optimization problem that maximizes the participant average safety level (PAS) for the entire network, where $P A S=\left(\sum_{i=1}^{N} S_{i}\right) / N$. Formally, given $P$ the set of participants with $|P|=N, T_{i, j}$ the trust level matrix, and $H=\left\{H^{(1)}, H^{(2)}, \ldots, H^{(M)}\right\}$ the set of EDHC that connect all the nodes, we want to find a better EDHC set $H^{\prime}=$ $\left\{H^{\prime(1)}, H^{\prime(2)}, \ldots, H^{\prime(M)}\right\}$ that maximizes PAS. 


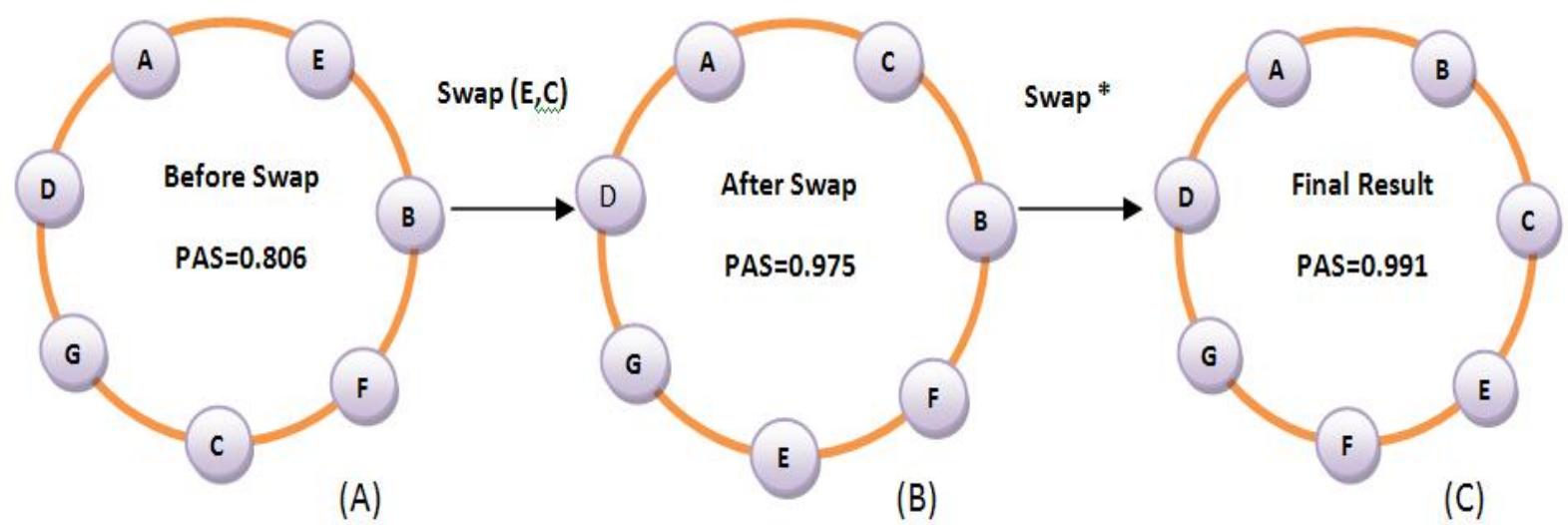

Figure 3.7: Swap Operation

\subsection{Greedy Algorithm for TE-HSMC}

Here, we present a greedy approximation algorithm, known as swap improvement (SI), for the optimization problem given in the previous section. The idea behind SI is quite simple; try to swap the position of a pair of participants in all HCs of $H$. If the swap provides an increase in PAS then save the modified $\mathrm{HC}$ set $H^{\prime}$ as $H$, otherwise discard the modified HC set $H^{\prime}$. Repeat swap operation on $H$ until there is no more improvement in PAS. Figure 3.7 provides a walk-through of SI for the example of Section 3.2.1 with trust levels given by Table 3.1. Figure 3.7(A) is the initial configuration. Figure 3.7(B) is the configuration after swapping the position of E and C. Continuing this approach, Figure 3.7(C) gives the final solution, since additional swaps don't help improve PAS. As can be seen here - and as noted in Section 3.2 - the new cycle $(\mathrm{C})$ provides more security against collusion.

\subsubsection{Experimental results}

We evaluate the performance of the greedy algorithm SI for TE-HSMC against a synthetic workload. We generate the trust level matrix, $T_{i, j}$, by simulating different network environments. We also randomly select an initial network configuration (a random HC). Then, we 
apply the greedy SI algorithm to improve PAS. The results of our experiments are given in Table 3.2 .

An explanation of Table 3.2 is in order. Column 1"(uniform distribution)," means trust level $T_{i, j}$ is chosen from uniform distribution, and "(N: m,d)" means trust level $T_{i, j}$ follows a normal distribution with the parameters $m$ (mean) and $d$ (standard deviation). I.PAS column gives the PAS for the initial network configuration (a random HC). F.PAS is the final PAS level after applying SI to the initial configuration. I.MINS is the minimum of all $S_{i}{ }^{\prime} s$ in the configuration while F.MINS is the minimum of all $S_{i}{ }^{\prime} s$ in the final configuration. I.MAX and F.MAXS can similarly be explained. Each row of Table 3.2 is the average value of 100 different trials.

Table 3.3 computes the improvement provided by SI, as a ratio of the before-and-after effect of the corresponding statistic. In most cases, SI offers a significant improvement in PAS, especially in environments with low trust level, for example, $N: m=0.25, d=0.125$.

\begin{tabular}{|c|c|c|c|c|c|c|}
\hline Test Case / Item & I.PAS & F.PAS & I.MINS & I.MAXS & F.MINS & F.MAXS \\
\hline "10 Participants (Uniform Distribution) & 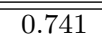 & 0.997 & 0.337 & $\overline{0.981}$ & 0.978 & 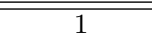 \\
\hline 20 Participants (Uniform Distribution) & 0.747 & 0.996 & 0.262 & 0.99 & 0.964 & 1 \\
\hline 50 Participants (Uniform Distribution) & 0.748 & 0.996 & 0.18 & 0.996 & 0.964 & 1 \\
\hline 100 Participants (Uniform Distribution) & 0.751 & 0.997 & 0.122 & 0.998 & 0.969 & 1 \\
\hline 10 Participants $(N: m=0.5, d=0.25)$ & 0.75 & 0.994 & 0.436 & 0.962 & 0.964 & 1 \\
\hline 20 Participants $(N: m=0.5, d=0.25)$ & 0.75 & 0.992 & 0.352 & 0.989 & 0.921 & 1 \\
\hline 50 Participants $(N: m=0.5, d=0.25)$ & 0.75 & 0.994 & 0.27 & 0.998 & 0.913 & 1 \\
\hline 100 Participants $(N: m=0.5, d=0.25)$ & 0.748 & 0.996 & 0.187 & 0.999 & 0.925 & 1 \\
\hline 10 Participants $(N: m=0.75, d=0.25)$ & 0.93 & 0.999 & 0.751 & 0.999 & 0.993 & 1 \\
\hline 20 Participants $(N: m=0.75, d=0.25)$ & 0.925 & 0.999 & 0.668 & 1 & 0.996 & 1 \\
\hline 50 Participants $(N: m=0.75, d=0.25)$ & 0.924 & 0.999 & 0.58 & 1 & 0.999 & 1 \\
\hline 100 Participants $(N: m=0.75, d=0.25)$ & 0.925 & 0.999 & 0.532 & 1 & 0.999 & 1 \\
\hline 10 Participants $(N: m=0.25, d=0.25)$ & 0.456 & 0.991 & 0.106 & 0.798 & 0.934 & 1 \\
\hline 20 Participants $(N: m=0.25, d=0.25)$ & 0.471 & 0.989 & 0.067 & 0.852 & 0.852 & 1 \\
\hline 50 Participants $(N: m=0.25, d=0.25)$ & 0.474 & 0.987 & 0.017 & 0.922 & 0.719 & 1 \\
\hline 100 Participants $(N: m=0.25, d=0.25)$ & 0.465 & 0.988 & 0.003 & 0.953 & 0.69 & 1 \\
\hline 10 Participants $(N: m=0.5, d=0.125)$ & 0.743 & 0.996 & 0.588 & 0.87 & 0.969 & 1 \\
\hline 20 Participants $(N: m=0.5, d=0.125)$ & 0.75 & 0.995 & 0.557 & 0.895 & 0.931 & 1 \\
\hline 50 Participants $(N: m=0.5, d=0.125)$ & 0.75 & 0.996 & 0.518 & 0.92 & 0.887 & 1 \\
\hline 100 Participants $(N: m=0.5, d=0.125)$ & 0.749 & 0.996 & 0.488 & 0.934 & 0.843 & 1 \\
\hline 10 Participants $(N: m=0.75, d=0.125)$ & 0.939 & 0.999 & 0.858 & 0.993 & 0.993 & 1 \\
\hline 20 Participants $(N: m=0.75, d=0.125)$ & 0.937 & 0.997 & 0.827 & 0.996 & 0.977 & 1 \\
\hline 50 Participants $(N: m=0.75, d=0.125)$ & 0.937 & 0.998 & 0.802 & 0.999 & 0.978 & 1 \\
\hline 100 Participants $(N: m=0.75, d=0.125)$ & 0.937 & 0.999 & 0.781 & 1 & 0.98 & 1 \\
\hline 10 Participants $(N: m=0.25, d=0.125)$ & 0.437 & 0.987 & 0.233 & 0.634 & 0.91 & 1 \\
\hline 20 Participants $(N: m=0.25, d=0.125)$ & 0.439 & 0.993 & 0.186 & 0.671 & 0.905 & 1 \\
\hline 50 Participants $(N: m=0.25, d=0.125)$ & 0.438 & 0.996 & 0.138 & 0.701 & 0.877 & 1 \\
\hline 100 Participants $(N: m=0.25, d=0.125)$ & 0.436 & 0.996 & 0.094 & 0.726 & 0.817 & 1 \\
\hline
\end{tabular}

Table 3.2: Synthetic Workload and Swap Improvement 


\begin{tabular}{|c|c|c|c|}
\hline Test Case / Item & F.PAS/I.PAS-1 & F.MINS/I.MINS-1 & F.MAXS/I.MAXS-1 \\
\hline 10 Participants (Uniform Distribution) & $34.548 \%$ & $190.21 \%$ & $1.94 \%$ \\
\hline 20 Participants (Uniform Distribution) & $33.33 \%$ & $267.94 \%$ & $1.01 \%$ \\
\hline 50 Participants (Uniform Distribution) & $33.16 \%$ & $435.56 \%$ & $0.40 \%$ \\
\hline 100 Participants (Uniform Distribution) & $32.76 \%$ & $694.26 \%$ & $0.20 \%$ \\
\hline 10 Participants $(N: m=0.5, d=0.25)$ & $32.53 \%$ & $121.10 \%$ & $3.95 \%$ \\
\hline 20 Participants $(N: m=0.5, d=0.25)$ & $32.27 \%$ & $161.65 \%$ & $1.11 \%$ \\
\hline 50 Participants $(N: m=0.5, d=0.25)$ & $32.53 \%$ & $238.15 \%$ & $0.20 \%$ \\
\hline 100 Participants $(N: m=0.5, d=0.25)$ & $33.16 \%$ & $394.65 \%$ & $0.10 \%$ \\
\hline 10 Participants $(N: m=0.75, d=0.25)$ & $7.42 \%$ & $32.22 \%$ & $0.10 \%$ \\
\hline 20 Participants $(N: m=0.75, d=0.25)$ & $8.00 \%$ & $49.10 \%$ & $0.00 \%$ \\
\hline 50 Participants $(N: m=0.75, d=0.25)$ & $8.12 \%$ & $72.24 \%$ & $0.00 \%$ \\
\hline 100 Participants $(N: m=0.75, d=0.25)$ & $8.00 \%$ & $87.78 \%$ & $0.00 \%$ \\
\hline 10 Participants $(N: m=0.25, d=0.25)$ & $117.33 \%$ & $781.13 \%$ & $25.31 \%$ \\
\hline 20 Participants $(N: m=0.25, d=0.25)$ & $109.98 \%$ & $1171.64 \%$ & $17.37 \%$ \\
\hline 50 Participants $(N: m=0.25, d=0.25)$ & $108.23 \%$ & $4129.41 \%$ & $8.46 \%$ \\
\hline 100 Participants $(N: m=0.25, d=0.25)$ & $112.47 \%$ & $22900.00 \%$ & $4.93 \%$ \\
\hline 10 Participants $(N: m=0.5, d=0.125)$ & $34.05 \%$ & $64.80 \%$ & $14.94 \%$ \\
\hline 20 Participants $(N: m=0.5, d=0.125)$ & $32.67 \%$ & $67.15 \%$ & $11.73 \%$ \\
\hline 50 Participants $(N: m=0.5, d=0.125)$ & $32.80 \%$ & $71.24 \%$ & $8.70 \%$ \\
\hline 100 Participants $(N: m=0.5, d=0.125)$ & $32.98 \%$ & $72.75 \%$ & $7.07 \%$ \\
\hline 10 Participants $(N: m=0.75, d=0.125)$ & $6.39 \%$ & $15.73 \%$ & $0.71 \%$ \\
\hline 20 Participants $(N: m=0.75, d=0.125)$ & $6.40 \%$ & $18.14 \%$ & $0.40 \%$ \\
\hline 50 Participants $(N: m=0.75, d=0.125)$ & $6.51 \%$ & $21.95 \%$ & $0.10 \%$ \\
\hline 100 Participants $(N: m=0.75, d=0.125)$ & $6.62 \%$ & $25.48 \%$ & $0.00 \%$ \\
\hline 10 Participants $(N: m=0.25, d=0.125)$ & $125.86 \%$ & $290.56 \%$ & $57.73 \%$ \\
\hline 20 Participants $(N: m=0.25, d=0.125)$ & $126.20 \%$ & $386.56 \%$ & $49.03 \%$ \\
\hline 50 Participants $(N: m=0.25, d=0.125)$ & $127.40 \%$ & $535.51 \%$ & $42.65 \%$ \\
\hline 100 Participants $(N: m=0.25, d=0.125)$ & $128.44 \%$ & $769.15 \%$ & $37.74 \%$ \\
\hline
\end{tabular}

Table 3.3: Performance of Swap Improvement 


\section{CHAPTER 4}

\section{ANONYMOUS OPT-OUT AND ID}

\section{ASSIGNMENT}

Anonymous opt-out (AOO) is a very useful tool in preventing statistical disclosure during the PPDM process. In this chapter, we will first introduce the idea of AOO. Then, we will discuss its implementation in PPDM. Two different anonymous ID assignment algorithms will be presented. Finally, we provide an analysis of these algorithms.

\subsection{Anonymous Opt-Out}

One problem in privacy preserving data mining schemes is statistical disclosure. We use an example to illustrate this issue. Consider a data mining network for retailers with 10 participants A, B, C, ... J. Suppose within these 10 participants, A is the only one who routinely sells a specific itemset, while others rarely sell (or sell very limited quantity). If we apply SS computation to count the global support value for this itemset, it may be easy for others to estimate A's contribution to the global sum. In fact, statistical disclosure can occur even in a $k$-collusion resistant algorithm, since we are still using SS computation. In order to solve this problem, Willenborg and Waal [49] suggest the use of AOO - an anonymous opt-out method - to prevent statistical disclosure for their distributed SMC. The idea behind AOO 
is to give any participant an opportunity to optionally withdraw its contribution from the SS computation. It does so by making the global sum void - without any other participant knowing who, if any, has withdrawn.

\subsubsection{Overview of Dining Cryptographers (DC) problem}

AOO lets all participants in the network know that the global sum is invalid if any participant opts-out by perhaps inputting an incorrect value as its contribution; however, we don't want anyone to know who has withdrawn. This is very similar to a very famous problem - Dining Cryptographers (DC) [16]. Suppose several cryptographers gather around a table for dinner. The DC problem is to design an algorithm that reveals who picks up the tab for the dinner party: the National Security Agency (NSA), or one of the cryptographers. If it is one of the cryptographers, its identity (of the cryptographer) is unknown to the other cryptographers. Actually, the solution to the DC problem can be viewed as a Boolean-OR SMC. Several methods have been developed in the past two decades to solve the DC problem effectively and securely. These methods are also known as anonymous veto algorithms.

The first solution to the DC problem, DC-Net, is given by Chaum [16]. DC-Net is an anonymous broadcast protocol. In DC-Net, every cryptographer first establishes a shared one-bit secret with his neighbor. Then, each cryptographer publicly announces a value. If he did not pick up the tab, this value is the Exclusive OR (XOR) of the shared bit he holds with his two neighbors, else (he picks up the tab, and) this value is the complement of the XOR. In Figure 4.1, cryptographer A and B share a secret bit 1, A and $\mathrm{C}$ share 0 , and $\mathrm{B}$ and $\mathrm{C}$ share 1. The first figure illustrates the situation when NSA pays the bill. The second figure shows the situation when A picks up the tab (A outputs the complement of 1 XOR 0). However, neither B nor $\mathrm{C}$ knows that cryptographer A paid the tab.

The DC-Net also provides provable security in the absence of trusted parties and the protocol is simple and elegant; however it has several limitations like collusion, disruption and protocol complexity. Many researchers have proposed variations to this basic protocol. 

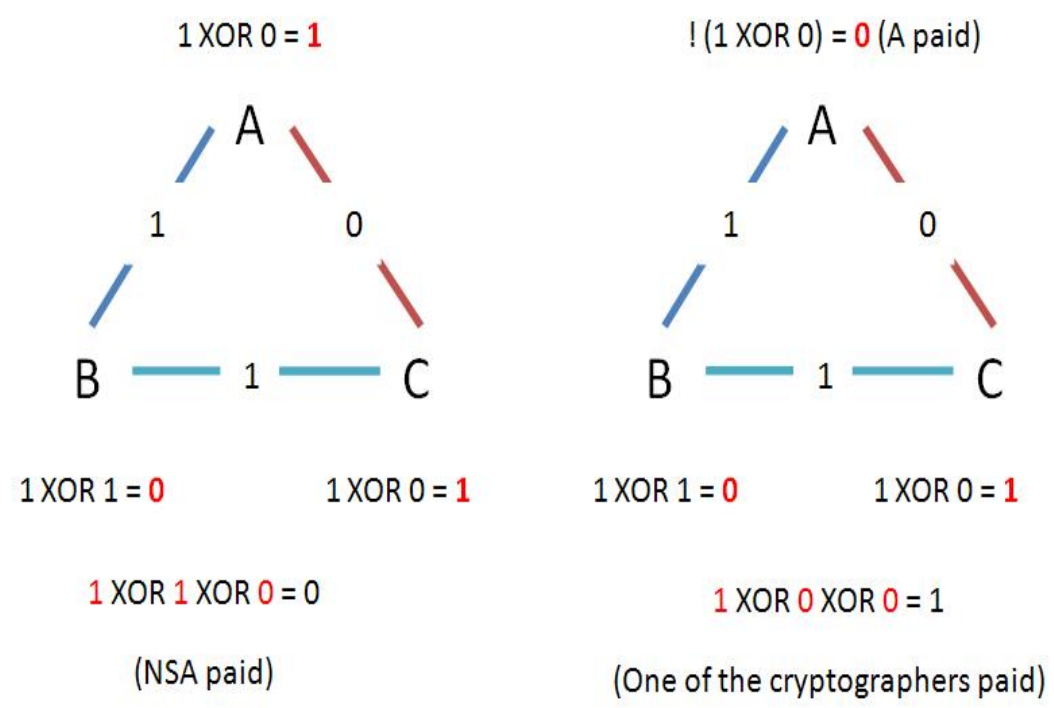

Figure 4.1: Illustration of DC-Net

Kiayias and Yung [30] investigate a Distributed Decision Making problem, and propose a 3-round broadcast veto protocol. However in their work, a third-party - a bulletin board server - is used to administer this process. Groth [24] modified Kiayias and Yung's work to reduce the complexity of the system. Their method requires $n+1$ rounds of broadcast where $n$ is the number of participants.

Recently, Hao and Zielinski [27] present an Anonymous Veto Protocol (AV-Net). The purpose of AV-Net is to overcome the limitations of DC-Net like collusion, disruption and complexity. AV-Net is also computationally secure under the Decision Diffie-Hellman (DDH) assumption. Let $\mathrm{G}$ denote a finite cyclic group of prime order q in which the DDH problem is intractable [11], and let $g$ be the generator. AV-Net works as follows. In round 1, each participant $p_{i}$ selects a random number $x_{i} \in Z_{q}$ and broadcasts $g^{x_{i}}$ together with a Zero-knowledge proof for the $x_{i}$. After round 1, each participant computes $g^{y_{i}}=\prod_{j=1}^{i-1} g^{x_{j}} / \prod_{j=i+1}^{n} g^{x_{j}}$. In round 2 , every participant $p_{i}$ broadcasts a value $g^{c_{i} y_{i}}$, where $c_{i}=x_{i}$, if $p_{i}$ wants to veto. If $p_{i}$ does not want to veto, it broadcasts $g^{c_{i} y_{i}}$ instead, where $r_{i}, c_{i}=r_{i}$ is a random number. At the end of round 2 , each $p_{i}$ computes $R=\prod_{i} g^{c_{i} y_{i}}$. If no one vetoes, $R=1$, else $R \neq 1$. 


\subsubsection{Anonymous opt-out in CPSS}

Shepard 39] presents an AOO algorithm using cycle-partitioned secure sum (CPSS). It computes the global sum in a secure fashion, provides collusion resistance, and allows a node to opt-out - or invalidate the global sum - in an anonymous manner.

Since the goal of AOO is to give participants an opportunity to veto or invalidate the result anonymously, the most straightforward way to implement AOO is to apply an anonymous veto algorithm - like AV-Net - before every SS computation. If no one vetoes, the following SS computation will be executed as normal. If some participants want to opt-out, then the following SS computation will be cancelled. The security of this process is promised by the anonymous veto algorithm one chooses. However this method is not efficient in CPSS from the complexity point of view. Actually, the best anonymous veto algorithm (AV-Net) needs 2 rounds of broadcast which is slower than computing the global sum of a single itemset in CPSS. In order to reduce the overhead of AOO, one can trade-off some security for improved performance. Instead of using an anonymous veto algorithm like AV-Net, we use a modified CPSS computation to implement the AOO part.

In CPSS computation, we have $n$ participants and each participant $p_{i}$ has a private input $x_{i}$ and we need to compute the $\sum_{i=1}^{n} x_{i}$ securely. In order to implement AOO, we ask each participant to retain another private input $y_{i}$ to express $p_{i}$ 's intention: whether $p_{i}$ is opting-in ( $x_{i}$ is valid) or opting-out to nullify the global sum ( $x_{i}$ is garbage). If $p_{i}$ is opting-in, $y_{i}$ is

0. On the other hand, $y_{i}$ is 1 , if $p_{i}$ is opting-out. As noted earlier, we do CPSS computation for $y_{i}$ 's as well. After using the CPSS algorithm to compute the $\sum_{i=1}^{n} y_{i}$, it is clear that $\sum_{i=1}^{n} y_{i} \geq 1$ if any one opts-out.

An example is given in Figure 4.2 and Figure 4.3, no one opts-out in Figure 4.2, while a node opts-out in Figure 4.3. In Figure 4.2, every participant has an input $y_{i}=0$ and we have the $\sum_{i=1}^{5} y_{i}=0$ at the end of the computation meaning that no participant has opted out. In Figure 4.3 , on the other hand, the node at the south-east corner opts-out by outputting $y_{i}=1$ and $x_{i}=29$ (a random number or garbage). Everyone will be notified that the result 
is invalid since $\sum_{i=1}^{5} y_{i}=1$.

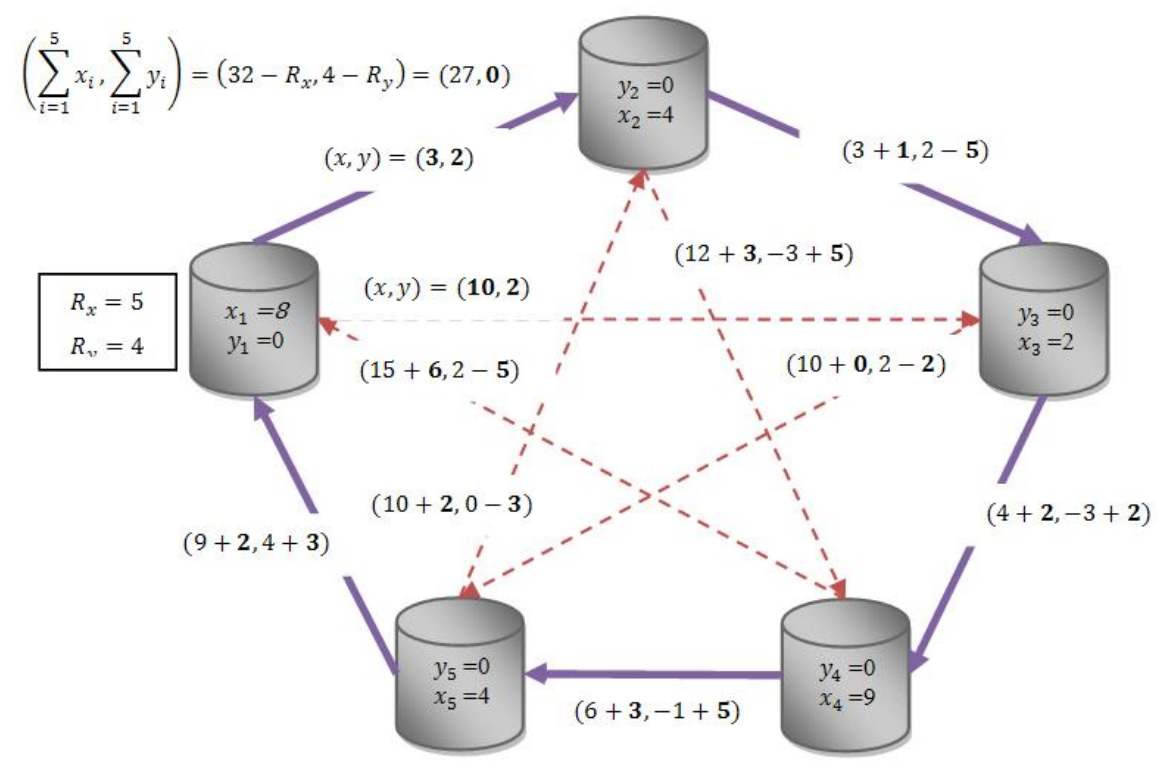

Figure 4.2: CPSS with Opt-Out (None Opts-out)

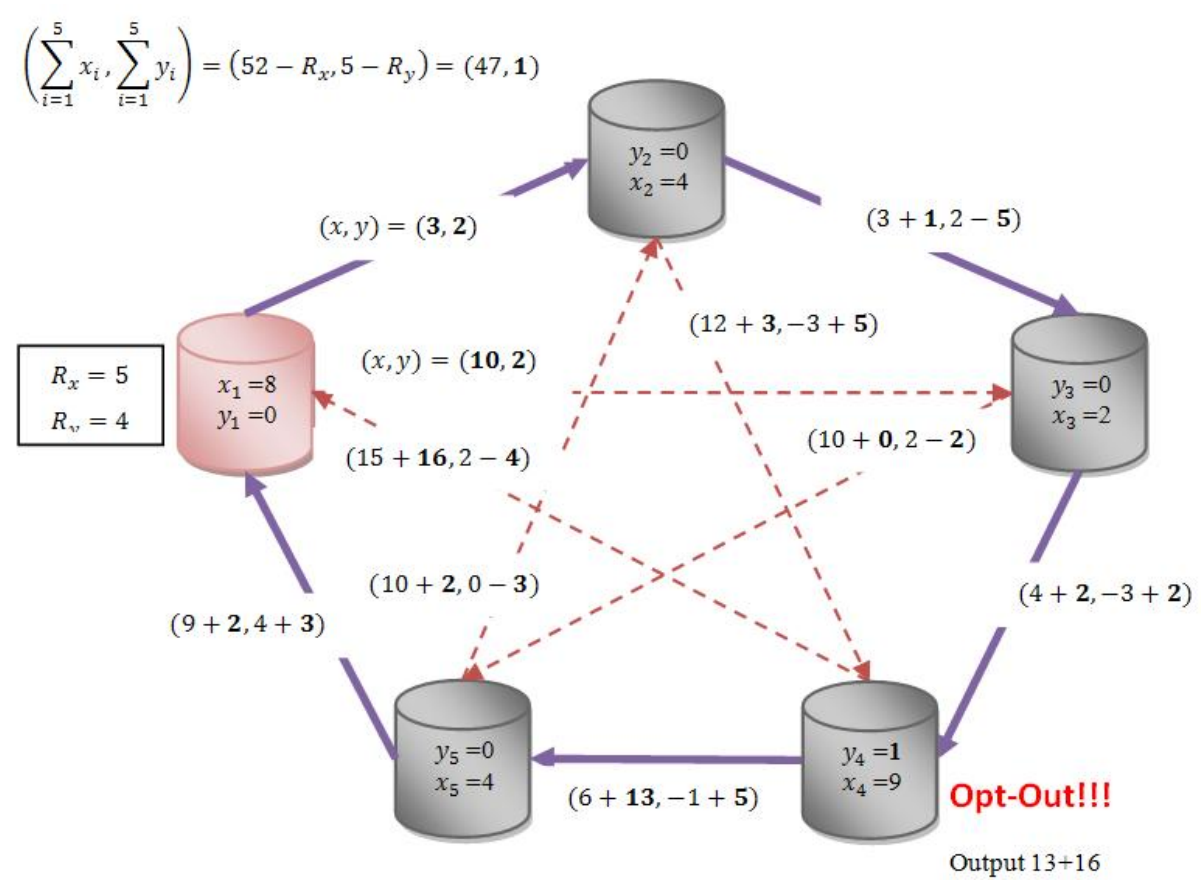

Figure 4.3: CPSS with Opt-Out (Someone Opts-out)

The AOO method we discussed above takes additional CPSS computation (y value) for 
each CPSS computation ( $\mathrm{x}$ value). It is believed to be more effective than using anonymous veto algorithms like AV-Net which needs 2 rounds of broadcast. However, using CPSS computation to implement AOO is less secure than anonymous veto algorithms, since security, if provided by CPSS computation, may be subject to collusion attack.

In the next section, we look at another problem, anonymous ID assignment.

\subsection{Anonymous ID}

One important goal of PPDM is to prevent disclosure of privacy information. However, it is possible in some cases we may want to identify each participant and use this information to administer the data mining process. For example, in AOO, we may wish to limit the number of times a participant is allowed to withdraw its result. It is impossible to handle this if the system cannot identify the participants. These problems can be addressed by assigning anonymous ID to nodes. The idea of anonymous ID is to give each participant in the system a unique, anonymous number (ID). Anonymous ID can be quite helpful in PPDM. For example, Shepard [39] presents a method to apply the idea of anonymous ID to the AOO process. By using this method, one can not only perform AOO successfully, but also collect some statistical information like the frequency of opt-out of each ID (though we do not know which ID belongs to whom, since they are anonymous). In the rest of this chapter, we will mainly focus on anonymous ID assignment algorithms.

\subsubsection{Anonymous ID assignment using SS}

The goal of anonymous ID assignment algorithms (AIDA) is to assign a set of unique IDs to each participant in a distributed system without disclosing which ID belongs to which participant (remain anonymous). Formally, given a set of participant $P$ and $|P|=n$, construct a function $f: P \rightarrow N^{+}$where $f\left(p_{i}\right) \neq f\left(p_{j}\right)(i \neq j)$, and $f\left(p_{i}\right)$ is unknown to all $p_{j}(i \neq j)$. 


\section{AIDA-A: Basic anonymous ID assignment algorithm}

Now, we present our first AIDA algorithm, AIDA-A. The algorithm has several rounds and each round has two steps.

Step 1: Each $p_{i}$ has an integer slot array $x_{i}=\left[x_{i, 1}, \ldots, x_{i, m}\right], m \geq|P|=n$. Set $x_{i, t_{i}}=1$ $\left(t_{i}\right.$ is the number that $p_{i}$ wants to choose as its anonymous ID and ID $=t_{i}$ must be available for selection) and $x_{i, k}=0, k \neq t_{i}$. Now, to provide collusion resistance, each $p_{i}$ splits its array $x_{i}$ into $n$ random arrays $x_{i}^{(j)}=\left[x_{i, 1}^{(j)}, \ldots, x_{i, m}^{(j)}\right]$, where $x_{i}=\left[\sum_{j=1}^{n} x_{i, 1}^{(j)}, \ldots, \sum_{j=1}^{n} x_{i, m}^{(j)}\right]$. Send array $x_{i}^{(j)}$ to $p_{j}$.

Step 2: Each participant $p_{j}$ adds all the $x_{i}^{(j)}$ from Step 1, and broadcasts the sum to everyone. It is easy to see that the sum of all these individual broadcasts equals $X=$ $\sum_{i=1}^{n} x_{i}=\left[X_{1}, \ldots, X_{m}\right]=\left[\sum_{i=1}^{n} x_{i, 1}, \ldots, \sum_{i=1}^{n} x_{i, m}\right]$. If $X_{t}=1$, it means only 1 participant selected $t$ as its ID and there is no conflict for ID $=t$. Therefore, this ID will be assigned to the one who selected it in Step 1 and is no longer available - for others - in future rounds. If $X_{t}>1$ or $X_{t}=0$, this means that either none or more than one participant selected $t$ as the ID, and ID $t$ is up for bid in future rounds.

For all $p_{i}$, whose ID selection has a conflict in Step 2, repeat Step 1 and 2 until everyone gets an ID without a conflict. However, as noted in the previous paragraph, nodes can only bid for IDs that have not already been assigned.

An example of the algorithm is given in Figure 4.4. After the first round of the algorithm, we have $X=\sum_{j=1}^{n} \sum_{i=1}^{n} x_{i}^{(j)}=[1,0,2]$ which indicates that two participants $\left(p_{1}\right.$ and $\left.p_{2}\right)$ wanted ID $=3$ as their anonymous ID. Therefore, both $p_{1}$ and $p_{2}$ will enter the next round of the algorithm. However, they can only select ID $=2$ or ID $=3$, since ID $=1$ has no conflict in the first round and has already been assigned to $p_{3}$ in round 1 .

One problem with the algorithm is that it may run forever. For example, it may never stop if two participants always try to select the same ID in every round. In order to solve this problem we present another algorithm which will give us a finite upper bound on the 

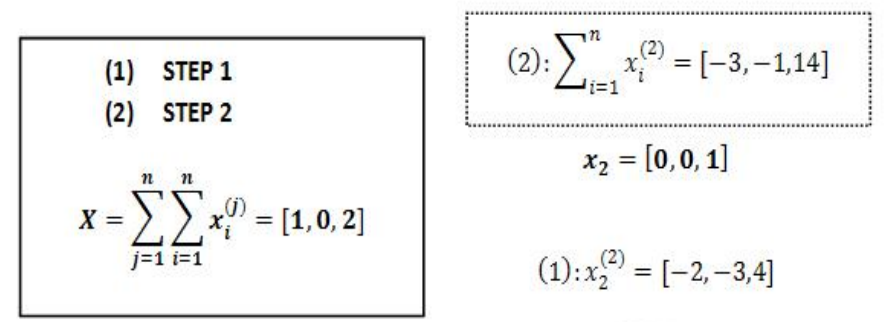

(1): $x_{2}^{(2)}=[-2,-3,4]$

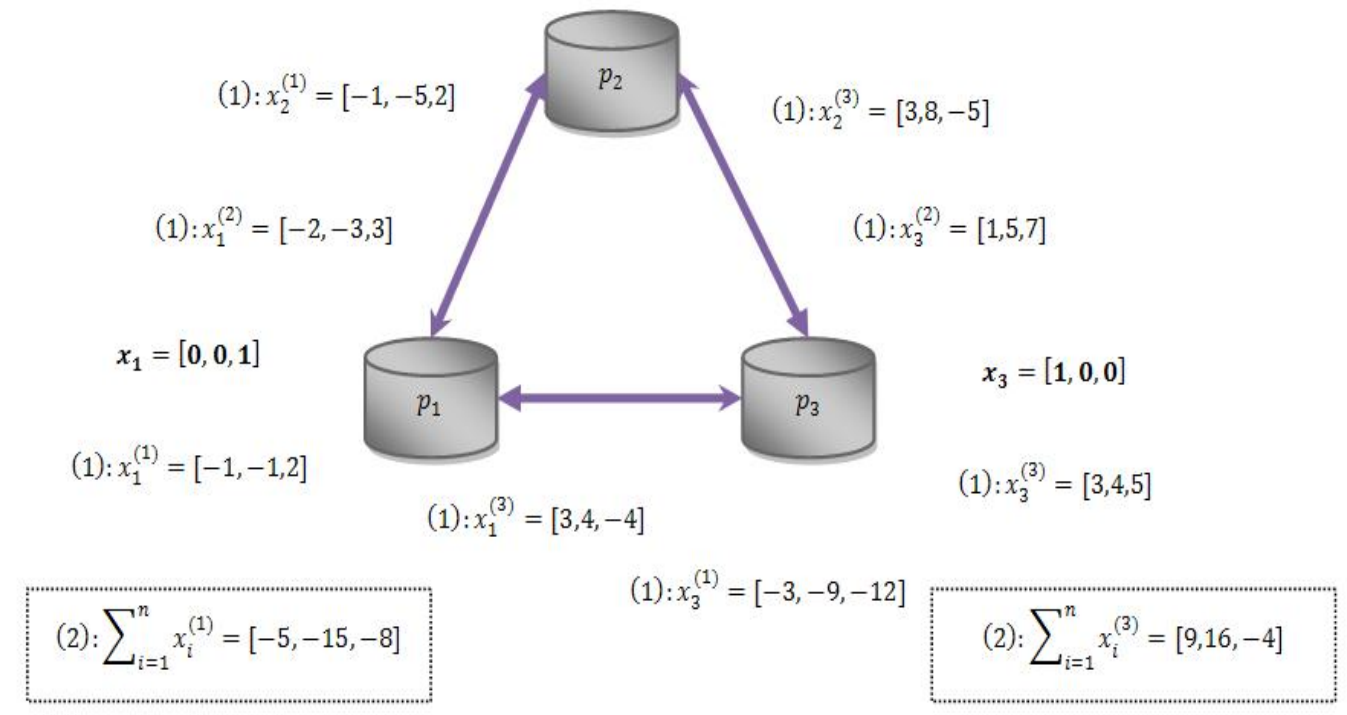

Figure 4.4: Basic Anonymous ID Assignment (AIDA-A), $m=3, n=3$

running time. We call the modified algorithm, AIDA-B. The idea behind AIDA-B is to assign at least one ID in each round even when there is a conflict.

\section{AIDA-B: Modified anonymous ID assignment algorithm}

Select a random participant $p_{s}$ as a source node and one $\mathrm{HC}$ in the system. The algorithm, just like AIDA-A, has several rounds and each round contains two steps (Figure 4.5). After we explain the details of the algorithm, we will connect it to Figure 4.5 .

Step 1: For each $p_{i},(i \neq s)$ construct an integer slot array $x_{i}=\left[x_{i, 1}, \ldots, x_{i, m}\right], m \geq|P|$, with $x_{i, t_{i}}=r\left(r>0\right.$ a random number, $t_{i}$ is the number that $p_{i}$ wants to choose as its anonymous ID), and $x_{i, k}=0$ for $k \neq t_{i}$.

Source node $p_{s}$ starts the computation by first creating a random slot $x_{s}=\left[x_{s, 1}, \ldots, x_{s, m}\right]$, 
and saves a copy locally in $\left[r_{s, 1}, \ldots, r_{s, m}\right]$. Node $p_{s}$ sends $x_{s}$ through the HC; each node adds its own $x_{i}$ to the received $x_{i}^{\prime}=\left[x_{i, 1}^{\prime}, \ldots, x_{i, m}^{\prime}\right]$ before forwarding to the next node. However, before adding $x_{i}$, each node $p_{i}$ saves a copy of the received $x_{i, t_{i}}^{\prime}$ - the value of $t_{i}^{\text {th }}$ element of the received slot array.

Step 2: $p_{s}$ receives a modified array $x_{s}^{\prime}=\left[\sum_{i=1}^{n} x_{i, 1}, \ldots, \sum_{i=1}^{n} x_{i, m}\right]$ after the SS computation. Let $X=x_{s}^{\prime}-x_{s}=\left[X_{1}, \ldots, X_{m}\right]$; if $X_{i}=0$, no one selected ID $=i$. On the other hand, if $X_{i}>0$, one or more nodes selected ID $=i$. Source node $p_{s}$ creates a new array $R=\left[R_{1}, \ldots, R_{m}\right]$ for broadcast to all the other nodes. $R_{i}$ is set to $x_{s, i}$ when $X_{i} \neq 0$. Else $R_{i}$ may be set to $x_{s, i}$ or to a different value. The broadcast is to let each node know if it got an ID or not. Every $p_{i}$ compares the saved $x_{i, t_{i}}^{\prime}$ to the incoming broadcast value $R_{t_{i}}$. If they are equal, $p_{i}$ is approved for using $t_{i}$ as its anonymous ID.

All participants without an anonymous ID assigned in the previous round will contend in the next round, carrying out Steps 1 and 2. This process repeats until each participant (except $p_{s}$ ) has an approved ID. In the end, $p_{s}$ selects an ID - which no one else selected as its own ID.

Figure 4.5 illustrates how this algorithm works. Node $p_{s}$ starts the computation; it chooses $x_{s}=\left[x_{s, 1}, \ldots, x_{s, m}\right]=[7,4,2,3,5,1]$, and sends a copy of this array $x_{s}$ (as $x_{2}^{\prime}=$ $[7,4,2,3,5,1])$ to next node $p_{2}$. Node $p_{2}$ chooses an ID, $t_{i}=3$, and constructs slot array $x_{2}=[0,0,2,0,0,0]$ with $t_{i}^{t h}$ slot set to 2 , i.e. $x_{2,3}=r=2(r=2$ is a random number $)$. It retains a copy of the $t_{i}^{\text {th }}$ value, $x_{2,3}^{\prime} . p_{2}$ adds $x_{2}$ to the incoming $x_{2}^{\prime}$ and forwards to next node, and so on. Finally, node $p_{s}$ receives $x_{s}^{\prime}=[7,4,7,3,9,1]$. It computes $X=x_{s}^{\prime}-x_{s}=$ $[0,0,5,0,4,0]$ and notes that slot 3 and $5($ ID $=3$ and ID $=5)$ are chosen by one or more nodes, since they are non-zero. In Step 2, it creates a modified array $R, R=[3,4, \mathbf{2}, 3, \mathbf{5}, 0]$ for broadcast to everyone; note that slot 3 and 5 are set to 2 and 5 (same as $x_{s, 3}$ and $x_{s, 5}$ ), while other slots - not chosen by anyone - are set to a random value. Node $p_{2}$, upon receving $R$, knows that it got the ID it asked for in Step 1, since $x_{2,3}^{\prime}=2=R_{3}$. However, $p_{4}$ is not as lucky as $p_{2}$ at getting an ID, since $x_{4,3}^{\prime}=4 \neq R_{3}=2$. 


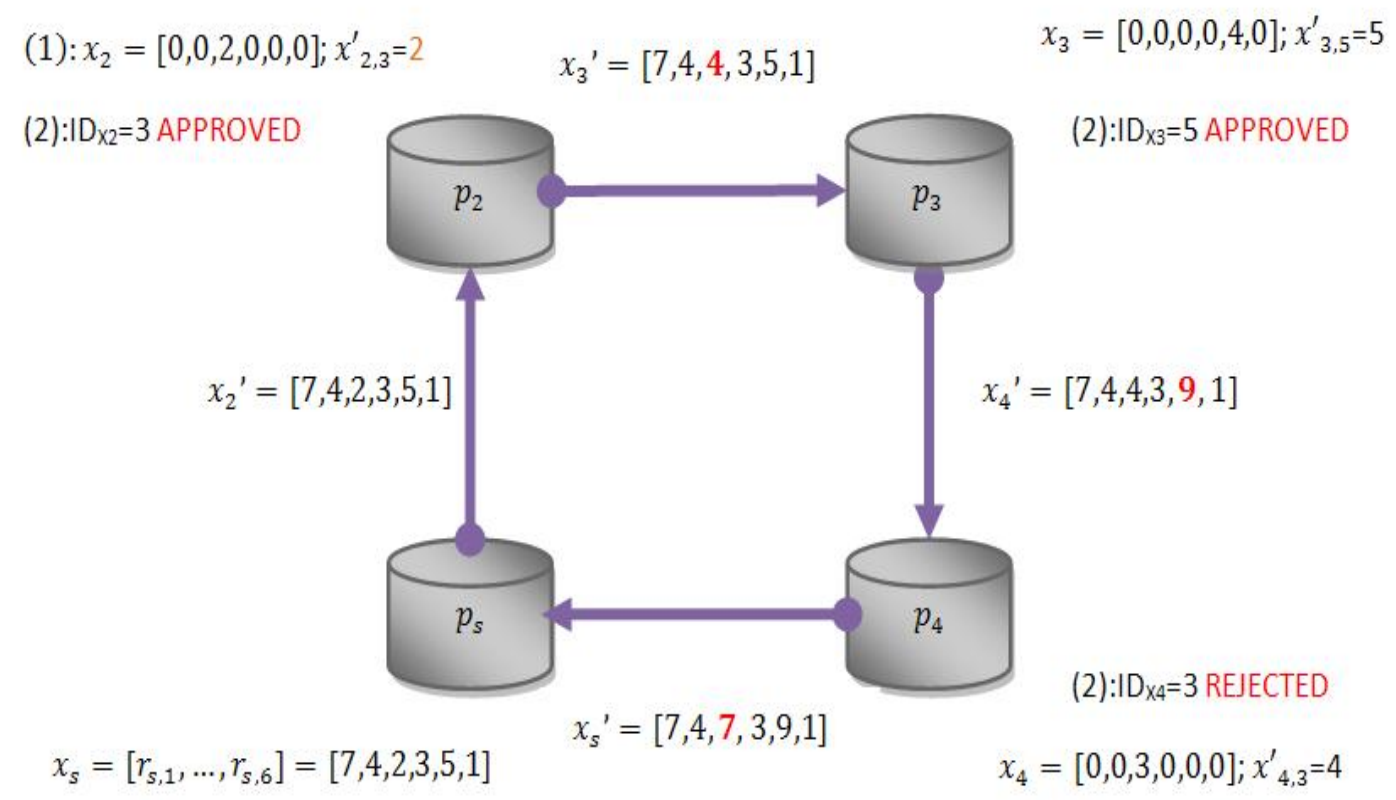

$$
\begin{aligned}
& \text { (1) } \text { Step } 1 \\
& \text { (2) } \text { Step } 2 \\
X= & x_{s}^{\prime}-x_{s}=[0,0,5,0,4,0] \\
R= & {[3,4,2,3,5,0] }
\end{aligned}
$$

Figure 4.5: Modified Anonymous ID Assignment (AIDA-B), $m=6, n=3$

\subsubsection{Analysis of anonymous ID algorithms}

In the previous section, we presented two anonymous ID assignment algorithms: AIDA-A and AIDA-B. These two algorithms are very similar to each other, except that AIDA-A will not assign an ID to anyone if more than one participant selects this ID; AIDA-B, on the other hand, will always assign the ID to the first participant who selects an ID. It is clear that the complexity of these two algorithms are dependent on how many rounds the algorithm needs before finishing the anonymous ID assignment. In this section, an analysis of these two algorithms is presented.

Let $N$ be the number of participants who need an anonymous ID and $M$ be the size of the slot array (or number of IDs) for both AIDA-A and AIDA-B. The goal of this analysis is to compute - given a fixed $M$ and $N$ - the number of rounds needed by the algorithm to 
complete the assignment. We divide our analysis into several small problems.

\section{Problem 1: Probability of finishing the algorithm in one round}

In order to finish the algorithm in one round, all participants have to select different IDs regardless of the algorithm we use (AIDA-A or AIDA-B). Given fixed $M$ and $N \leq M$, we can easily derive the following equation.

$$
\operatorname{Prob}_{(\text {AIDA finish in one round) }}=\frac{\left(\begin{array}{l}
M \\
N
\end{array}\right) N !}{M^{N}}=\frac{M !}{(M-N) ! M^{N}}=\prod_{i=1}^{N-1}\left(1-\frac{i}{M}\right)
$$

An approximation to Equation (4.1) can be obtained using the Taylor's formula.

$$
\begin{aligned}
e^{x} & =1+\frac{x}{1 !}+\frac{x^{2}}{2 !}+\frac{x^{3}}{3 !}+\ldots, \quad-\infty \leq x \leq+\infty \\
& \approx 1+x
\end{aligned}
$$

Replacing $1-\frac{i}{M}$ in Equation 4.1 with $e^{-\frac{i}{M}}$ leads to

$$
\operatorname{Prob}_{(\text {AIDA finish in one round) }} \approx \prod_{i=1}^{N-1} e^{-\frac{i}{M}}=e^{-\sum_{i=1}^{N-1} \frac{i}{M}}=e^{-\frac{N(N-1)}{2 M}}
$$

Equation (4.2) can be used to answer two questions. Given fixed $M$ and $p \in[0,1]$, what is the maximum value for $M$ such that the probability of finishing AIDA in one round is at least $p$ ? Using Equation 4.2 we have

$$
\begin{aligned}
p & \leq e^{-\frac{N(N-1)}{2 M}}, \\
0 & \leq N^{2}-N-2 M \ln \frac{1}{p} \\
N & \leq \frac{1}{2}+\sqrt{\frac{1}{2}+2 M \ln \frac{1}{p}}
\end{aligned}
$$

Given fixed $N$ and $p \in[0,1]$, what is the minimum value for $M$ such that the probability of 
finishing AIDA in one round is at least p? Again, using Equation (4.2) we have

$$
\begin{gathered}
p \leq e^{-\frac{N(N-1)}{2 M}}, \\
M \geq \frac{N^{2}-N}{2 \ln \frac{1}{p}} .
\end{gathered}
$$

\section{Problem 2: Number of IDs assigned in one round}

Now that we know the probability of finishing the assignment algorithm in one round, let us consider an extension of the previous problem: how many IDs can be assigned in one round? Here we denote by $D$ the number of anonymous IDs assigned in a single round.

We first look at AIDA-A algorithm. Here, if more than one participant selects the same ID, the ID will not be assigned to anyone. We first consider the number of combination of assigning $M^{\prime}$ IDs to $N^{\prime}$ participants, where each ID is selected by none, or more than two participants. This number is denoted by $C_{(A I D A-A)}\left(M^{\prime}, N^{\prime}\right)$.

Construct a generating function

$$
G(x)=\left(1+\frac{x}{1 !}+\frac{x^{2}}{2 !}+\frac{x^{3}}{3 !}+\ldots\right)^{M^{\prime}}
$$

Replace $1+\frac{x}{1 !}+\frac{x^{2}}{2 !}+\frac{x^{3}}{3 !}+\ldots$ in Equation 4.5 with $e^{x}-x$ by applying the Taylor's formula.

$$
\begin{aligned}
G(x) & =\left(e^{x}-x\right)^{M^{\prime}} \\
& =\sum_{i=0}^{M^{\prime}}\left(\begin{array}{c}
M^{\prime} \\
i
\end{array}\right)(-1)^{i} x^{i} e^{x\left(M^{\prime}-i\right)} \\
& =\sum_{i=0}^{M^{\prime}}\left(\begin{array}{c}
M^{\prime} \\
i
\end{array}\right)(-1)^{i} x^{i} \sum_{r=0}^{\infty} \frac{1}{r !}\left(M^{\prime}-i\right)^{r} x^{r} .
\end{aligned}
$$

Define $C_{(A I D A-A)}\left(M^{\prime}, N^{\prime}\right)$ to be $N^{\prime} ! \times\left(\right.$ coefficient of $x^{N^{\prime}}$ in $\left.G(x)\right)$ where,

$$
\text { coefficient of } x^{N^{\prime}} \operatorname{in} G(x)=\sum_{i=0}^{N^{\prime}}\left(\begin{array}{c}
M^{\prime} \\
i
\end{array}\right)(-1)^{i} \frac{1}{\left(N^{\prime}-i\right) !}\left(M^{\prime}-i\right)^{N^{\prime}-i}
$$


Thus,

$$
C_{A I D A-A}\left(M^{\prime}, N^{\prime}\right)=N^{\prime} ! \sum_{i=0}^{N^{\prime}}\left(\begin{array}{c}
M^{\prime} \\
i
\end{array}\right)(-1)^{i} \frac{1}{\left(N^{\prime}-i\right) !}\left(M^{\prime}-i\right)^{N^{\prime}-i}
$$

Given $M$ IDs and $N$ participants, the number of combination of assigning $D$ anonymous IDs to $N$ participants without conflicts is given by

$$
\left(\begin{array}{c}
M \\
D
\end{array}\right)\left(\begin{array}{c}
M \\
D
\end{array}\right) D ! C_{A I D A-A}(M-D, N-D)
$$

Given $M$ IDs and $N$ participants, the probability of assigning $D$ anonymous IDs without conflict in one round is given by

$$
\operatorname{Prob}_{(A I D A-A)}(D, M, N)=\frac{\left(\begin{array}{c}
M \\
D
\end{array}\right)\left(\begin{array}{c}
M \\
D
\end{array}\right) D ! C_{A I D A-A}(M-D, N-D)}{M^{N}}
$$

Now we can easily compute the expected value of the number of anonymous ID assigned in one round for AIDA-A.

$$
\begin{aligned}
\operatorname{Expected}_{(A I D A-A)}(M, N) & =\sum_{d=0}^{N} \operatorname{Prob}_{(A I D A-A)}(d, M, N) \cdot d \\
& =\sum_{d=0}^{N} \frac{\left(\begin{array}{c}
M \\
d
\end{array}\right)\left(\begin{array}{c}
M \\
d
\end{array}\right) d ! C_{A I D A-A}(M-d, N-d)}{M^{N}} \cdot d .
\end{aligned}
$$

In Figure 4.6, we plot the values of $\operatorname{Expected}_{(A I D A-A)}(M, N)$ for $0 \leq M \leq 50, N \leq 30$. For example, we can see from Figure 4.6 , given $M=50$ and $N=30$, we assign around 17 IDs, without conflict, in one round of AIDA-A. However, we can see from Equation 4.9) that the computation of $\operatorname{Expected}_{(A I D A-A)}(M, N)$ is complicated for large $M$ and $N$. In the following, we provide an approximation function, $\operatorname{ApExpected}_{(A I D A-A)}(M, N)$, to approximately represent Equation 4.9. There are two major advantages in finding an approximate function. The first one is that it is much faster to compute $\operatorname{ApExpected}_{(A I D A-A)}(M, N)$ compared to Expected $_{(A I D A-A)}(M, N)$. Another one is that the approximation converts the 


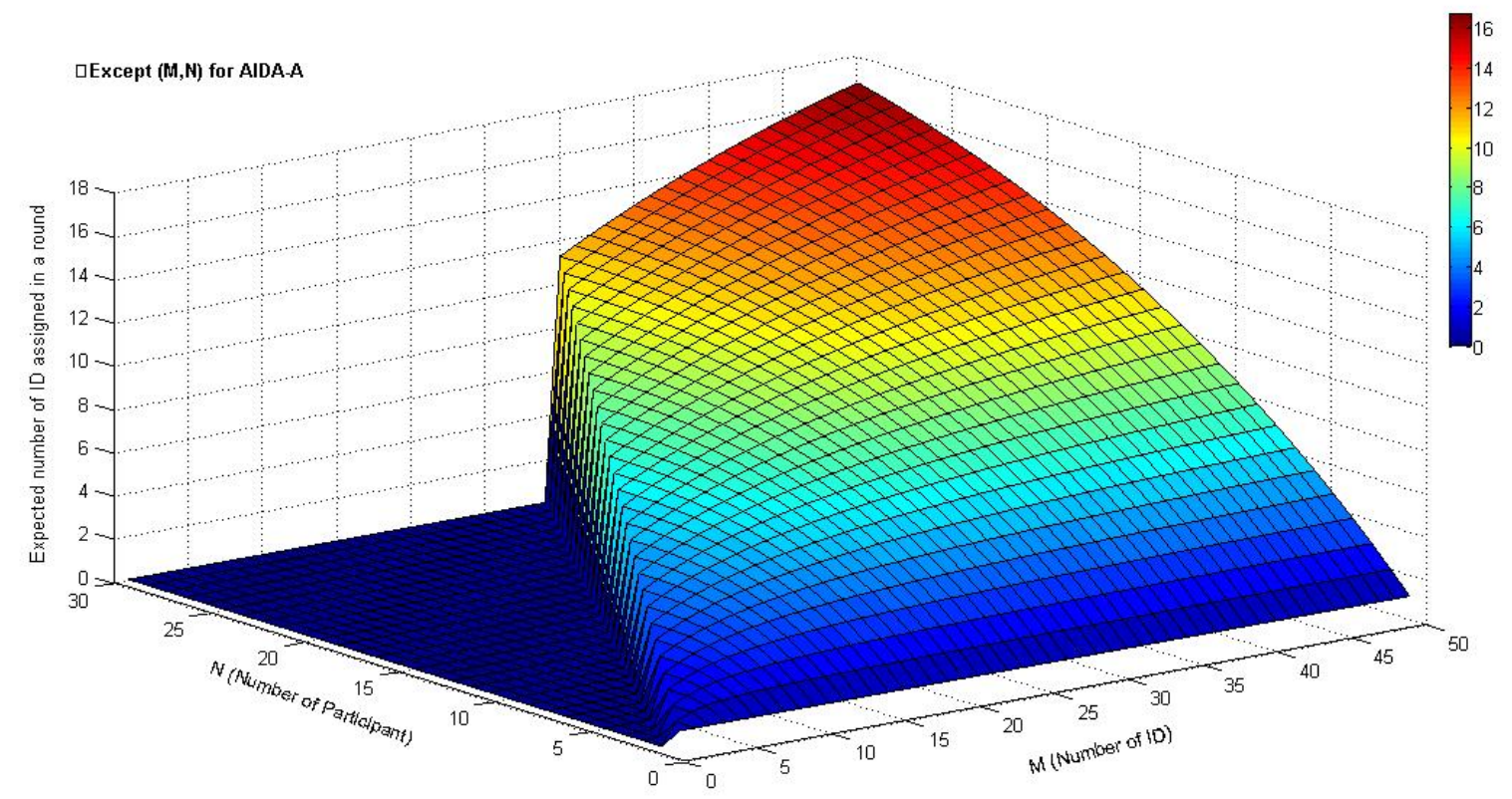

Figure 4.6: Mean Number of IDs Assigned in a Round (AIDA-A), $0 \leq M \leq 50, N \leq 30$

discrete function Expected $_{(A I D A-A)}(M, N)$ into a continuous function.

We use the least squares method to find $\operatorname{ApExpected}_{(A I D A-A)}(M, N)$ that minimizes Equation 4.10) below:

$$
\sum_{n=1}^{N} \sum_{m=1}^{M}\left(\operatorname{ApExpected}_{(A I D A-A)}(M, N)-\operatorname{Expected}_{(A I D A-A)}(M, N)\right)^{2}
$$

We use a bi-variate quadratic function, Equation (4.11), as a basis of our computation.

$$
\operatorname{ApExpected}_{(A I D A-A)}(M, N)=A m+B n+C m n+D m^{2}+E n^{2}+F .
$$

Solving this regression Equation (4.11) yields the following:

$$
\begin{aligned}
\text { ApExpected }_{(A I D A-A)}(M, N)= & 0.405 m+0.093 n+0.012 m n \\
& -0.014 m^{2}-0.002 n^{2}-0.044 .
\end{aligned}
$$




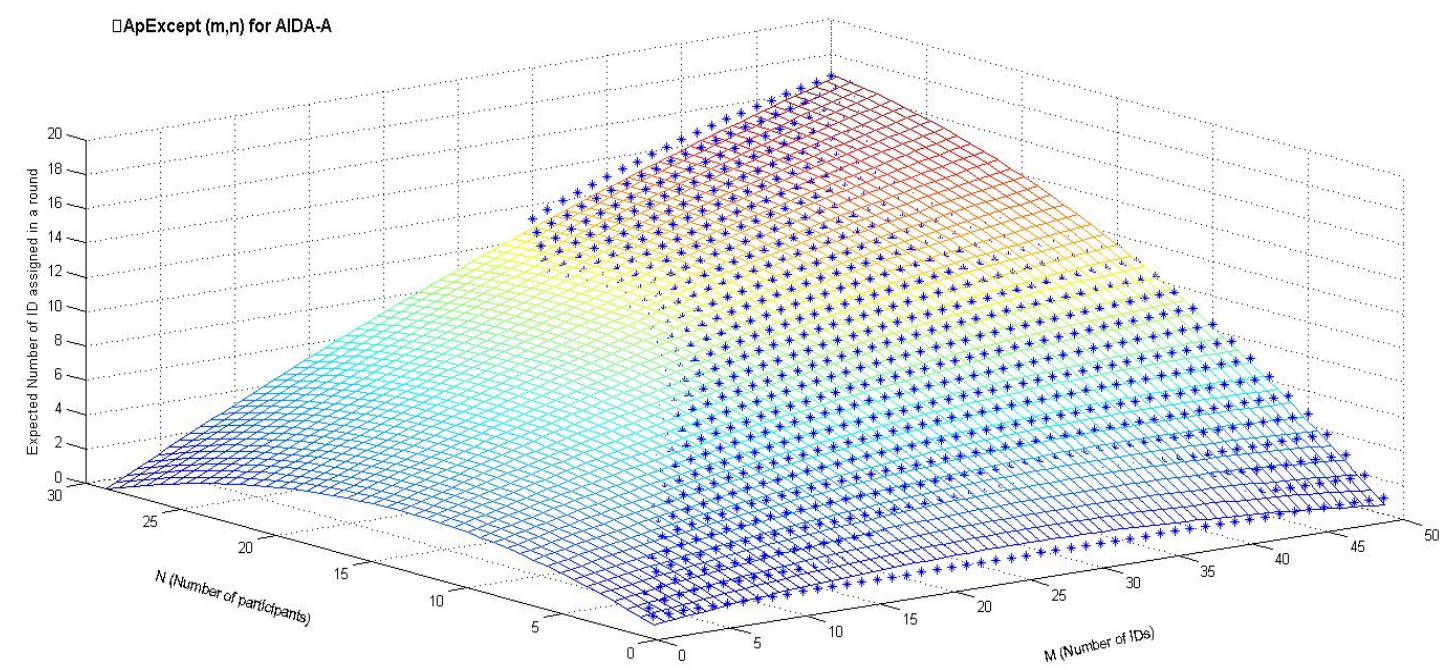

Figure 4.7: Comparison of $A_{p} \operatorname{Expected}_{(A I D A-A)}(M, N)$ and $\operatorname{Expected}_{(A I D A-A)}(M, N)$

In Figure 4.7, we plot the value of our approximate function $\operatorname{ApExpected}_{(A I D A-A)}(M, N)$, and the original function $\operatorname{Expected}_{(A I D A-A)}(M, N)$. The star in Figure 4.7 represents the value of $\operatorname{Expected}_{(A I D A-A)}(M, N)$ and the mask of the plot represents the value of $\operatorname{ApExpected}_{(A I D A-A)}(M, N)$. We can see from Figure 4.7 that $\operatorname{ApExpected}_{(A I D A-A)}(M, N)$ matches Expected $_{(A I D A-A)}(M, N)$ very well.

Now, we turn our attention to the analysis of AIDA-B. First, we compute the number of IDs assigned in one round for AIDA-B. AIDA-B assigns an ID to the first participant who selects that ID. We use the same steps, as in AIDA-A, to analyze AIDA-B.

We first consider the number of combination of assigning $M^{\prime}$ IDs to $N^{\prime}$ participants, where each ID has been selected by one or more participants. This number is denoted by $C_{(A I D A-B)}\left(M^{\prime}, N^{\prime}\right)$. Construct a generating function, $G(x)$ where

$$
G(x)=\left(x+\frac{x}{1 !}+\frac{x^{2}}{2 !}+\frac{x^{3}}{3 !}+\ldots\right)^{M^{\prime}} .
$$


Replace $x+\frac{x}{1 !}+\frac{x^{2}}{2 !}+\frac{x^{3}}{3 !}+\ldots$ in Equation 4.13 with $e^{x}-1$ by applying the Taylor's formula.

$$
\begin{aligned}
G(x) & =\left(e^{x}-1\right)^{M^{\prime}} \\
& =\sum_{i=0}^{M^{\prime}}\left(\begin{array}{c}
M^{\prime} \\
i
\end{array}\right)(-1)^{i} e^{x\left(M^{\prime}-i\right)} \\
& =\sum_{i=0}^{M^{\prime}}\left(\begin{array}{c}
M^{\prime} \\
i
\end{array}\right)(-1)^{i} \sum_{r=0}^{\infty} \frac{1}{r !}\left(M^{\prime}-i\right)^{r} x^{r} \\
& =\sum_{r=0}^{\infty}\left(\sum_{i=0}^{M^{\prime}}\left(\begin{array}{c}
M^{\prime} \\
i
\end{array}\right)(-1)^{i}\left(M^{\prime}-i\right)^{r}\right) \frac{1}{r !} x^{r} .
\end{aligned}
$$

It can be easily shown that $C_{(A I D A-B)}\left(M^{\prime}, N^{\prime}\right)=N^{\prime} ! \cdot\left(\right.$ the coefficient of $x^{N^{\prime}}$ in $\left.G(x)\right)$, where the coefficient of $x^{N^{\prime}}$ in $G(x)$ equals $\frac{1}{N^{\prime} !} \sum_{i=0}^{M^{\prime}}\left(\begin{array}{c}M^{\prime} \\ i\end{array}\right)(-1)^{i} M^{\prime}-i^{N^{\prime}}$. Thus,

$$
\begin{aligned}
C_{(A I D A-B)}\left(M^{\prime}, N^{\prime}\right) & =\sum_{i=0}^{M^{\prime}}\left(\begin{array}{c}
M^{\prime} \\
i
\end{array}\right)(-1)^{i}\left(M^{\prime}-i\right)^{N^{\prime}} \\
& =N ! \cdot S\left(M^{\prime}, N^{\prime}\right) .
\end{aligned}
$$

where, $S\left(M^{\prime}, N^{\prime}\right)=\frac{1}{N^{\prime} !} \sum_{i=0}^{M^{\prime}}\left(\begin{array}{c}M^{\prime} \\ i\end{array}\right)(-1)^{i}\left(M^{\prime}-i\right)^{N^{\prime}}$ is the Stirling Number of Second Kind [19]. Given $M$ IDs and $N$ participants, the number of combination of assigning $D$ anonymous ID to $N$ participants is

$$
\left(\begin{array}{c}
M \\
D
\end{array}\right) \cdot C_{(A I D A-B)}(D, N)
$$

And, the probability of assigning $D$ anonymous ID in one round is given by

$$
\operatorname{Prob}_{(A I D A-B)}(D, M, N)=\frac{\left(\begin{array}{c}
M \\
D
\end{array}\right) C_{(A I D A-B)}(D, N)}{M^{N}}
$$

Now we can derive the expected value of the number of anonymous ID assigned in one round 
for AIDA-B.

$$
\begin{aligned}
\operatorname{Expected}_{(A I D A-B)}(M, N) & =\sum_{d=0}^{N} \operatorname{Prob}_{(A I D A-B)}(D, M, N) \cdot d \\
& =\sum_{d=0}^{N} \frac{\left(\begin{array}{c}
M \\
d
\end{array}\right) C_{(A I D A-B)}(d, N)}{M^{N}} \cdot d .
\end{aligned}
$$

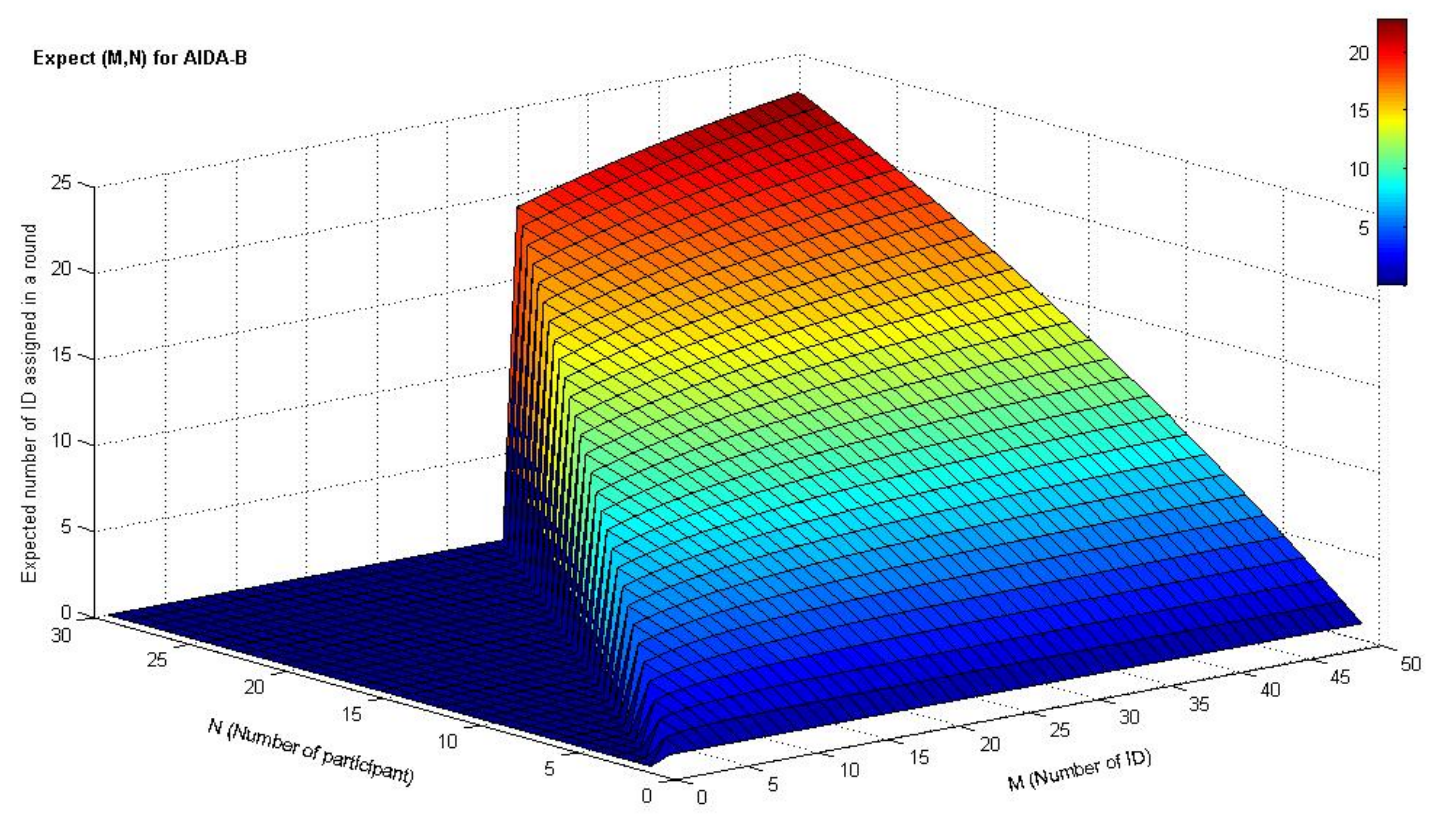

Figure 4.8: Mean Number of IDs Assigned in a Round (AIDA-B), $0 \leq M \leq 50, N \leq 30$

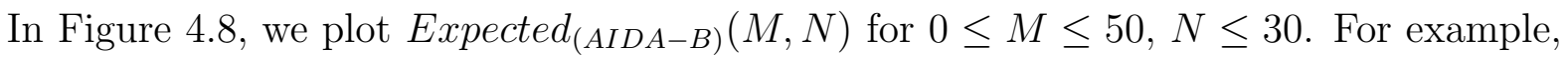
we can see from Figure 4.8 , given $M=50$ and $N=30$, we assign around 23 IDs in one round all without conflict. Compared to Figure 4.6, it is clear that AIDA-B assigns more anonymous IDs to the participants in a round than AIDA-A.

Just like the approximation Equation 4.12 for $\operatorname{Expected}_{(A I D A-A)}(M, N)$, we also find a bi-variate quadratic function $A p \operatorname{Expected}_{(A I D A-B)}(M, N)$ to approximate $\operatorname{Expected}_{(A I D A-B)}(M, N)$. 
By using the same method as before, we obtain the expression below.

$$
\begin{aligned}
\text { ApExpected }_{(A I D A-B)}(M, N)= & 0.662 m+0.055 n+0.007 m n \\
& -0.009 m^{2}-0.001 n^{2}-0.006 .
\end{aligned}
$$

\section{Problem 3: Number of rounds needed for the assignment}

Now that we have continuous approximate functions, for AIDA-A and AIDA-B, and the expected number of ID assigned in a round, we are ready to compute the (approximate) mean number of rounds needed for the two algorithms. Given $N$ and $M \geq N$, we use $A p \operatorname{ExpRound}(M, N)$ to approximately represent the expected value for the number of rounds, ApExpRound $(M, N)$, needed by the two algorithms.

$$
\operatorname{ApExpRound}(M, N)=\left\{\begin{array}{ll}
1+A p \operatorname{ExpRound}(M-E(M, N), N-E(M, N)) & \text { if } N>0 \\
0 & \text { if } N \leq 0
\end{array}\right\} .
$$

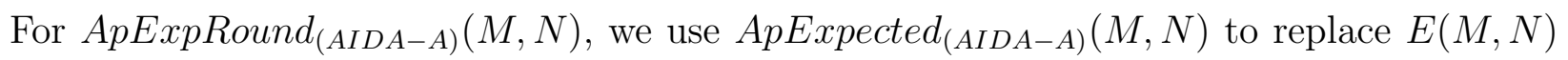
in Equation (4.19). However, for $A p \operatorname{ExpRound}(A I D A-B)(M, N)$, we use the expression for ApExpected $_{(A I D A-B)}(M, N)$ to replace $E(M, N)$ in Equation (4.19).

In Figure 4.9, we plot $A p \operatorname{ExpRound}(M, N)$ for both AIDA-A and AIDA-B. Red or light squares represent the value of $\operatorname{ApExpRound}_{(A I D A-A)}(M, N)$ - algorithm AIDA-A - and the blue or dark stars represent the value of $\operatorname{ApExpected}_{(A I D A-B)}(M, N)$ for AIDA-B. For example, given $M=N=30$, we can expect AIDA-A to finish its assignment in 7 rounds. However, AIDA-B is expected to finish faster, in 4 rounds. 


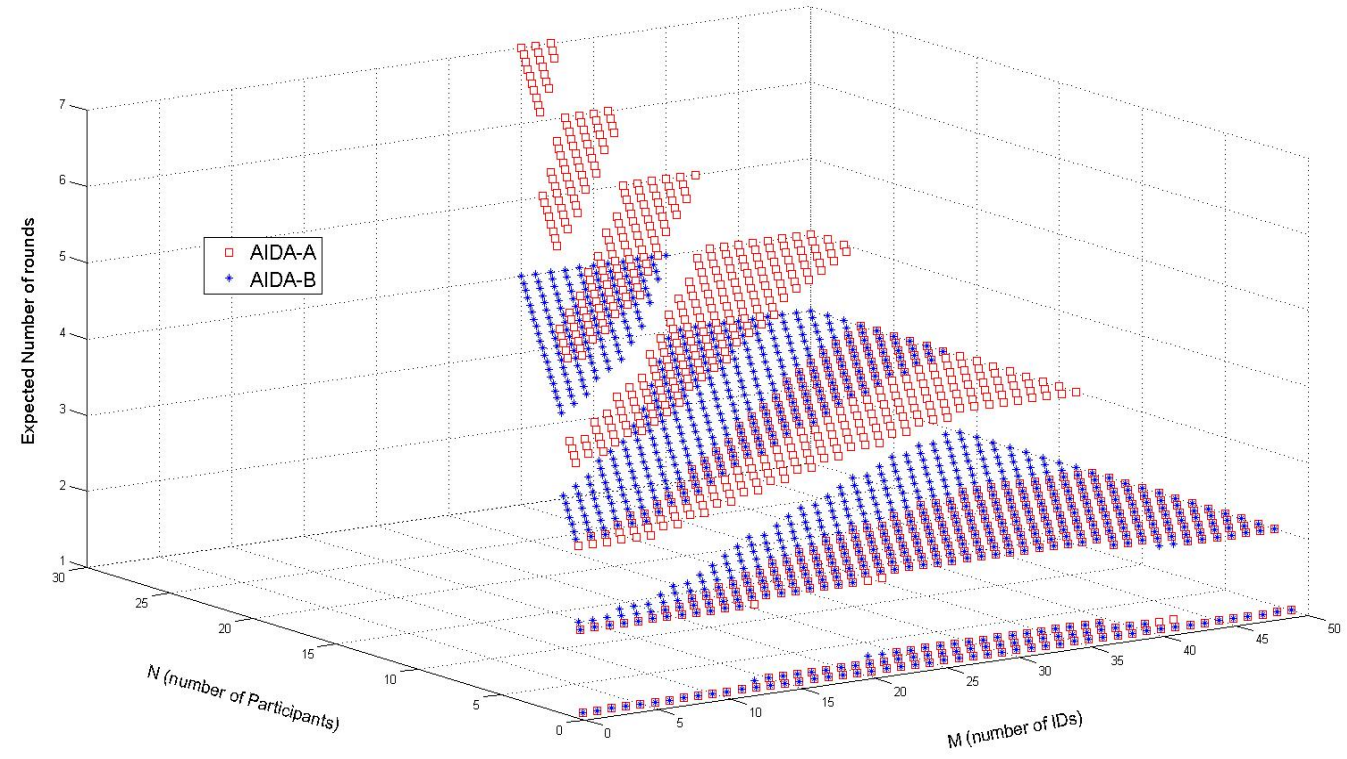

Figure 4.9: Approximate Evaluation of Mean Number of Rounds (AIDA-A and AIDA-B), $0 \leq M \leq 50, N \leq 30$ 


\section{CHAPTER 5}

\section{CONCLUDING REMARKS}

In this chapter, we recap our contributions and provide some pointers for future work.

\subsection{Contribution}

This thesis addressed four topics which are closely related to secure multiparty computation (SMC): edge-disjoint Hamiltonian cycle (EDHC), trust enabled SMC, anonymous opt-out, and anonymous ID assignment.

We first presented an interesting property of EDHC - sequence dependence, meaning that the sequence of finding EDHC in random graph can affect the number of EDHC one can possibly find. In order to explain the sequence dependent property of EDHC, we introduced a new problem - restricted edge-disjoint cycle (REDC), and proved that REDC is NPcomplete. The NP-completeness of REDC provides a basis for the sequence dependent property of EDHC. Based on this consideration, we established a connection between EDHC and another problem, maximum clique (MC). Then, we presented a heuristic dynamic local search algorithm for solving EDHC based on the dynamic local search algorithm for MC.

We introduced the notion of trust in HC based SMC. We presented a generalized model of trust and provided a greedy algorithm to improve the mean network safety level. Experimentation of the greedy algorithm using synthetic workload showed good improvements 
in the mean network safety. We also examined the problem of anonymous opt-out and anonymous ID assignment. We gave two options to implement anonymous opt-out in SMC - using anonymous veto algorithm, and using a modified secure sum computation. We presented a variant of an existing anonymous ID algorithm, and carried out an approximate mathematical analysis of these two algorithms.

\subsection{Future Work}

Our work used REDC to explain the sequence dependent property of EDHC. Others may want to examine the computational complexity of EDHC, under the assumption that an oracle machine is used to discover the underlying HC. It will be interesting to further develop the notion of trust introduced in this thesis and possibly improve upon the behavior of the greedy algorithm. Note that, the model we presented is only useful for SMC algorithms, which use $\mathrm{HC}$ as basis. A related research question is the notion of trust to a more generalized model which can be applied to any current SMC system - whether or not it uses HC. 


\section{BIBLIOGRAPHY}

[1] D. Agrawal and C.C. Aggarwal. On the design and quantification of privacy preserving data mining algorithms. In Proceedings of the twentieth ACM SIGMOD-SIGACTSIGART symposium on Principles of database systems, pages 247-255. ACM New York, NY, USA, 2001.

[2] R. Agrawal and R. Srikant. Fast algorithms for mining association rules in large databases. In Proceedings of the 20th International Conference on Very Large Data Bases, pages 487-499. Morgan Kaufmann Publishers Inc. San Francisco, CA, USA, 1994.

[3] B. Alspach, JC Bermond, and D. Sotteau. Decomposition into cycles 1: Hamilton decompositions. Cycles and Rays, page 9, 1990.

[4] D. Angluin and L.G. Valiant. Fast probabilistic algorithms for Hamiltonian circuits and matchings. In Proceedings of the ninth annual ACM symposium on Theory of computing, pages 30-41. ACM New York, NY, USA, 1977.

[5] MZ Ashrafi, D. Taniar, and K. Smith. ODAM: An optimized distributed association rule mining algorithm. IEEE Distributed Systems Online, 5(3), 2004.

[6] MM Bae and B. Bose. Edge disjoint Hamiltonian cycles in k-ary n-cubes and hypercubes. IEEE Transactions on Computers, 52(10):1271-1284, 2003. 
[7] R. Battiti and M. Protasi. Reactive local search for the maximum clique problem. Algorithmica, 2001.

[8] R. Battiti and G. Tecchiolli. The reactive tabu search. INFORMS Journal on Computing, 6(2):126, 1994.

[9] B. Bollobas, T.I. Fenner, and A.M. Frieze. An algorithm for finding Hamilton paths and cycles in random graphs. Combinatorica, 7(4):327-341, 1987.

[10] JA Bondy and US Murty. Graph Theory with Applications. The Macmillan Press LTD, 1976.

[11] D. Boneh. The decision Diffie-Hellman problem. Lecture Notes in Computer Science, 1423:48-63, 1998.

[12] C. Borgelt. Keeping things simple: finding frequent item sets by recursive elimination. In Proceedings of the 1st international workshop on open source data mining: frequent pattern mining implementations, pages 66-70. ACM New York, NY, USA, 2005.

[13] A.Z. Broder, A.M. Frieze, and E. Shamir. Finding hidden Hamiltonian cycles. Random Structures and Algorithms, 5(3), 1994.

[14] F. Brunacci. DB 2 and DB 2 A: Two useful tools for constructing Hamiltonian circuits. European Journal of Operational Research, 34(2):231-236, 1988.

[15] S. Busygin. A new trust region technique for the maximum weight clique problem. Discrete Applied Mathematics, 154(15):2080-2096, 2006.

[16] D. Chaum. The dining cryptographers problem: Unconditional sender and recipient untraceability. Journal of Cryptology, 1(1):65-75, 1988.

[17] DW Cheung, J. Han, VT Ng, AW Fu, and Y. Fu. A fast distributed algorithm for mining association rules. In Parallel and Distributed Information Systems, 1996., Fourth International Conference on, pages 31-42, 1996. 
[18] C. Clifton, M. Kantarcioglu, J. Vaidya, X. Lin, and M.Y. Zhu. Tools for privacy preserving distributed data mining. ACM SIGKDD Explorations Newsletter, 4(2):28-34, 2002.

[19] D.I.A. Cohen. Basic Techniques of Combinatoral Theory. New York etc, 1978.

[20] R. Diestel. Graph theory. Springer, 2005.

[21] AM Frieze. Finding Hamilton cycles in sparse random graphs. Journal of combinatorial theory. Series B, 44(2):230-250, 1988.

[22] M.R. Garey, D.S. Johnson, et al. Computers and Intractability: A Guide to the Theory of NP-completeness. W.H. freeman San Francisco, 1979.

[23] A. Grosso, M. Locatelli, and F.D. Croce. Combining swaps and node weights in an adaptive greedy approach for the maximum clique problem. Journal of Heuristics, $10(2): 135-152,2004$.

[24] J. Groth. Efficient maximal privacy in boardroom voting and anonymous broadcast. Lecture Notes in Computer Science, pages 90-104, 2004.

[25] J. Han and M. Kamber. Data mining: concepts and techniques. Morgan Kaufmann, 2006.

[26] J. Han, J. Pei, Y. Yin, and R. Mao. Mining frequent patterns without candidate generation: A frequent-pattern tree approach. Data Mining and Knowledge Discovery, $8(1): 53-87,2004$.

[27] F. Hao and P. Zielinski. A 2-round anonymous veto protocol. In Proceedings of the 14th International Workshop on Security Protocols, SPW, volume 6.

[28] Y. Hu and B. Panda. A data mining approach for database intrusion detection. In Proceedings of the 2004 ACM symposium on Applied computing, pages 711-716. ACM New York, NY, USA, 2004. 
[29] K. Katayama, A. Hamamoto, and H. Narihisa. Solving the maximum clique problem by k-opt local search. In Proceedings of the 2004 ACM symposium on Applied computing, pages 1021-1025. ACM New York, NY, USA, 2004.

[30] A. Kiayias and M. Yung. Non-interactive zero-sharing with applications to private distributed decision making. Lecture notes in computer science, pages 303-320, 2003.

[31] W. Kocay. An extension of the multi-path algorithm for finding Hamilton cycles. Discrete Mathematics, 101(1-3):171-188, 1992.

[32] W. Kocay and P.C. Li. An algorithm for finding a long path in a graph. Utilitas Mathematica, 45:169-185, 1994.

[33] J. Komlós and E. Szemerédi. Limit distribution for the existence of Hamiltonian cycles in a random graph. Discrete Mathematics, 306(10-11):1032-1038, 2006.

[34] A. Machanavajjhala, J. Gehrke, D. Kifer, and M. Venkitasubramaniam. l-diversity: Privacy beyond k-anonymity. In Proc. of ICDE, pages 24-35, 2006.

[35] J.S. Park, M.S. Chen, and P.S. Yu. An effective hash-based algorithm for mining association rules. In Proceedings of the 1995 ACM SIGMOD international conference on Management of data, pages 175-186. ACM New York, NY, USA, 1995.

[36] L. Posa. Hamiltonian circuits in random graphs. Discrete Math, 14(4):359-364, 1976.

[37] W. Pullan and H.H. Hoos. Dynamic local search for the maximum clique problem. Journal of Artificial Intelligence Research, 25:159-185, 2006.

[38] B. Schneier and W. Diffie. Applied cryptography: protocols, algorithms, and source code in C. 1996.

[39] S.S. Shepard. Anonymous Opt-Out and Secure Computation in Data Mining. Master's thesis, Bowling Green State University, 2007. 
[40] J.A. Shufelt and H.J. Berliner. Generating Hamiltonian circuits without backtracking from errors. Theoretical Computer Science, 132(1):347-375, 1994.

[41] C. Solnon and S. Fenet. A study of aco capabilities for solving the maximum clique problem. Journal of Heuristics, 12(3):155-180, 2006.

[42] J. Srivastava, R. Cooley, M. Deshpande, and P.N. Tan. Web usage mining: Discovery and applications of usage patterns from web data. SIGKDD explorations, 1(2):12-23, 2000 .

[43] I.A. Stewart. Distributed algorithms for building Hamiltonian cycles in k-ary n-cubes and hypercubes with faulty links. Journal of Interconnection Networks, 8(3):253, 2007.

[44] A. Thomason. A simple linear expected time algorithm for finding a hamilton path. In Graph Theory and Combinatorics, 1988: Proceedings of the Cambridge Combinatorial Conference in Honour of Paul Erdős, page 373. North Holland, 1989.

[45] S. URABE, J. WANG, E. KODAMA, and T. TAKATA. A Collusion-Resistant Approach to Privacy-Preserving Distributed Data Mining. IPSJ SIG Technical Reports, 2005(63):21-25, 2005.

[46] S. Urabe, J. Wang, E. Kodama, and T. Takata. A high collusion-resistant approach to distributed privacy-preserving data mining. IPSJ Digital Courier, 3(0):442-455, 2007.

[47] V.S. Verykios, E. Bertino, I.N. Fovino, L.P. Provenza, Y. Saygin, and Y. Theodoridis. State-of-the-art in privacy preserving data mining. ACM Sigmod Record, 33(1):50-57, 2004.

[48] DB West. Graph Theory. New Jersey: Prentice Hall, 1996.

[49] L. Willenborg and T. De Waal. Statistical disclosure control in practice. Springer, 1996. 
[50] A.C.C. Yao. How to generate and exchange secrets. In SFCS '86: Proceedings of the 27th Annual Symposium on Foundations of Computer Science, pages 162-167, Washington, DC, USA, 1986. IEEE Computer Society. 